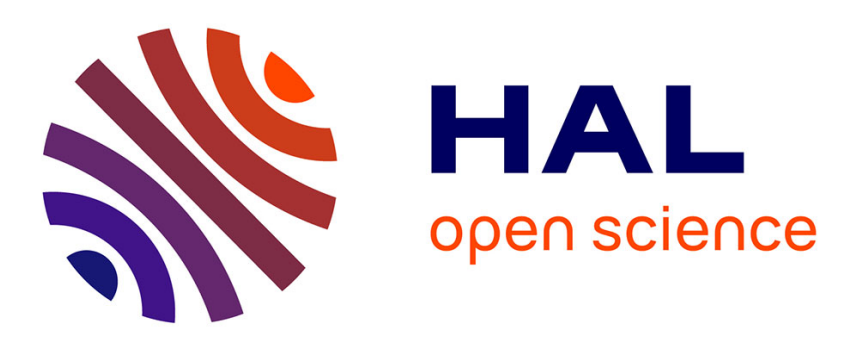

\title{
Saturation resonances by magnetic mode crossing in optical pumping with a multimode gas laser
}

M. Dumont

\section{To cite this version:}

M. Dumont. Saturation resonances by magnetic mode crossing in optical pumping with a multimode gas laser. Journal de Physique, 1972, 33 (11-12), pp.971-991. 10.1051/jphys:019720033011-12097100 . jpa-00207346

\section{HAL Id: jpa-00207346 https://hal.science/jpa-00207346}

Submitted on 1 Jan 1972

HAL is a multi-disciplinary open access archive for the deposit and dissemination of scientific research documents, whether they are published or not. The documents may come from teaching and research institutions in France or abroad, or from public or private research centers.
L'archive ouverte pluridisciplinaire HAL, est destinée au dépôt et à la diffusion de documents scientifiques de niveau recherche, publiés ou non, émanant des établissements d'enseignement et de recherche français ou étrangers, des laboratoires publics ou privés. 


\title{
" SATURATION RESONANCES BY MAGNETIC MODE CROSSING IN OPTICAL PUMPING WITH A MULTIMODE GAS LASER »
}

\author{
M. DUMONT
}

Laboratoire de Spectroscopie Hertzienne de l'ENS, 24, rue Lhomond, Paris 5e, France

(Reçu le 17 mai 1972)

\begin{abstract}
Résumé. - La lumière de fluorescence émise par des atomes $(\mathrm{Ne})$ pompés optiquement par un laser multimode présente des résonances lorsque l'écart Zeeman est égal à l'écart de fréquence entre modes. L'étude expérimentale montre que ces résonances sont sensibles à la phase relative des modes et que leur forme est voisine de celle de l'effet Hanle. Nous en donnons ici l'interprétation théorique à l'aide d'un calcul de perturbation. Deux effets peuvent produire des résonances : 1) Un effet de population (PE) qui est résonnant lorsque la composante $\sigma^{+}$d'un mode et la composante $\sigma^{-}$d'un autre mode interagissent avec des atomes de même vitesse (croisement de " trous »). La largeur de ces résonances est $2 \Gamma_{a b}^{\prime}$ (largeur naturelle de la raie élargie par collisions). 2) Un effet de cohérence Zeeman (ZCE) : l'action d'une paire de modes, $\left(\nu, \sigma^{+}\right)$et $\left(\mu, \sigma^{-}\right)$, crée (au $2^{\mathrm{e}}$ ordre) de l'alignement transversal modulé à la fréquence $\omega_{v}-\omega_{\mu}$ et résonnant quand l'écart Zeeman est égal à cette fréquence; par interaction avec une nouvelle paire de modes $\left(\lambda, \sigma^{-}\right)$et $\left(\kappa, \sigma^{+}\right)$ $\left(\omega_{\nu}-\omega_{\mu}+\omega_{\lambda}-\omega_{\kappa}=0\right)$, cet alignement donne (au $4^{\mathrm{e}}$ ordre) des grandeurs longitudinales non modulées, présentant en fonction du champ magnétique une résonance de largeur voisine de celle de l'effet Hanle $\left(\Gamma_{b}(2)\right)$.

Dans les conditions de nos expériences (modes rapprochés : $\Gamma_{a b}^{\prime} \gtrsim \Delta \omega>\Gamma_{b}(2)$ ), les résonances du premier type ne sont pas résolues et l'on n'observe que celle du second type qui sont très sensibles à la phase des modes. Nous discutons également la possibilité de mesurer des facteurs de Landé à l'aide de ces résonances.
\end{abstract}

\begin{abstract}
The fluorescence light emitted by atoms (Ne) optically pumped with a multimode laser beam exhibits a resonance each time the Zeeman splitting is equal to the frequency difference between two modes. The experimental study shows that these resonances are very sensitive to the relative phase of modes and that their width is approximately equal to the Hanle effect width. A theoretical interpretation is given with the help of a perturbation calculation. Two phenomena produce resonances : 1) A Population Effect (PE) which is resonant when the $\sigma^{+}$component of one mode and the $\sigma^{-}$component of another interact with atoms of same velocity (hole crossing). The width of these resonances is $2 \Gamma_{a b}^{\prime}$ (natural width broadened by collisions). 2) A Zeeman Coherence Effect (ZCE) : the action of a pair of modes $\left(v, \sigma^{+}\right)$and $\left(\mu, \sigma^{-}\right)$produces (at 2nd order) transverse alignment modulated at the beat frequency $\omega_{\nu}-\omega_{\mu}$ and resonant when the Zeeman splitting is equal to that frequency; by interaction with a second pair of modes $\left(\lambda, \sigma^{-}\right)$ and $\left(\kappa, \sigma^{+}\right)\left(\omega_{\nu}-\omega_{\mu}+\omega_{\lambda}-\omega_{\kappa}=0\right)$, this alignment produces unmodulated longitudinal quantities (4th order) which exhibit, as the magnetic field is scanned, a resonance whose width is close to that of the Hanle curve $\left(\Gamma_{b}(2)\right)$.

In our experimental conditions (close modes : $\Gamma_{a b}^{\prime} \gtrsim \Delta \omega>\Gamma_{b}(2)$ ), the resonances of the first kind are not resolved and the observed resonances are only of the second type's which arevery sensitive to the phases of modes. We also discuss the possibility of using these resonances to measure Landé $g$ factors.
\end{abstract}

I. Introduction. - The study of the fluorescence light emitted by excited atoms optically pumped by a laser beam provides a wide field of experiments. Many of them, dealing with atomic physics and spectroscopy (Hanle effect [1], magnetic resonances...) do not use the specific properties of the laser beam which is only a convenient light source. Nevertheless, besides some non linear effects which appears in the above experiments (such as power broadening of the Hanle effect), the spectral features of the laser beam can produce characteristic phenomena : for instance, with a monomode pumping beam, the Lamb dip can be observed on the fluorescence light [2]. With a multimode laser beam, the fluorescence light exhibits modulations [3], [4] at the beat frequencies between modes, which are resonant when their frequency is equal to the Zeeman splitting (this effect is linear with respect to the laser intensity). For the same values of the magnetic field, resonances appear on the non modulated part of the fluorescence light 
(first observed by Fork and al. [3]). These resonances are interpreted as a non linear effect due to the reciprocal saturation of the $\sigma^{+}$component of one mode by the $\sigma^{-}$component of another (the laser beam being linearly $\sigma$ polarized). They can be called saturation resonances by magnetic mode crossing.

The aim of this paper is to study theoretically these saturation resonances in order to explain some surprising experimental observations (Fig. 1 shows the experimental set up) : first, all resonances, except that in zero magnetic field, are very sensitive to the relative phase of modes. As shown on figure 2, all

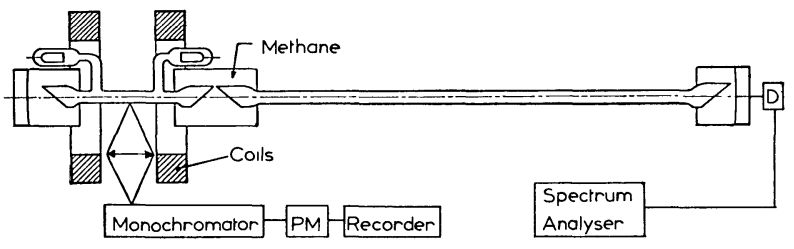

Fig. 1. - Experimental apparatus : the cell, filled with an $\mathrm{He}-\mathrm{Ne}$ mixture, is put inside the laser cavity and is submitted to an axial magnetic field. The fluorescence light, detected with a monochromator and a photomultiplier, is recorded as a function of the magnetic field. The laser oscillation can be obtained on several lines $\left(6328 \AA, 3 s_{2}-2 p_{4} ; 6401 \AA\right.$, $3 s_{2}-2 p_{2} ; 7305 \AA, 3 s_{2}-2 p_{1} ; 1.15 \mu, 2 s_{2}-2 p_{4} ; 1.52 \mu$, $2 s_{2}-2 p_{1} ; 3.39 \mu, 3 s_{2}-3 p_{4}$, without methane). The beat frequencies between modes are detected with the fast detector $D$ and measured with a spectrum analyser calibrated with a reference oscillator. The ratio between this beat frequency and saturation resonances spacing on the recorder provides a measurement of the Landé $g$ factors. The observation of the fine spectrum of each beat frequency allows one to determine if the modes are phase-locked (strong sharp monochromatic beat) or if they are free running (many unstable little components of slightly different frequencies).

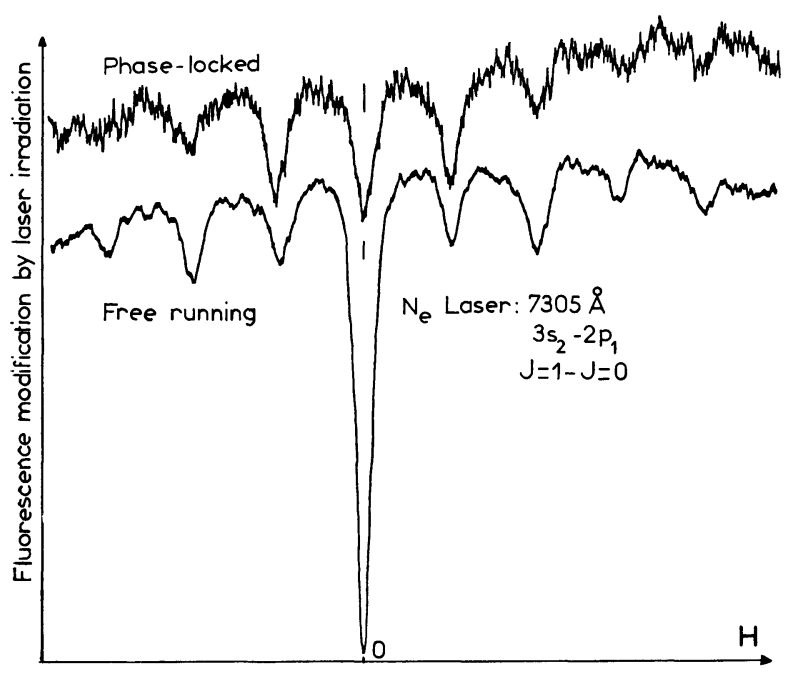

Fig. 2. - Experimental records of the saturation signal on the fluorescence lines. As the detection is made with a $\pi$ analyser the resonance in zero magnetic field is not the Hanle effect; like all lateral resonances, it can be explained only by non linear interactions (at least fourth order in the laser field). To obtain a spontaneously phase locked oscillation we must decrease the laser intensity. Therefore the intensity of the phase locked signal is much less than that of the free-running signal. Nevertheless these curves clearly demonstrate the difference of behaviour between the lateral resonances and the central one. resonances are of comparable amplitude when the modes are phaselocked; on the contrary, when the modes are free running the lateral resonances are much smaller than the central one. Secondly, the width of the resonances is of the order of magnitude of the Hanle effect width i. e. of the relaxation rate of alignment in the atomic levels. The intuitive interpretation, the crossing of two Bennett holes [5] in the population velocity distribution, cannot explain these two features : indeed the holes are phase insensitive and their width is much too large [6], [29]. Finally, a calculation is needed to determine the position of resonance when both levels have a Zeeman structure. Indeed, if it is possible to ascribe the resonances to one of the levels, it is easy to measure the Landé $g$ factor of this level by simultaneous measurement of the magnetic field for each resonance and of the beat frequencies between modes.

To calculate the intensity and the polarization of the fluorescence lines we have only to determine the average density matrix

$$
\bar{\rho}(r, t)=\int \rho(v, r, t) \mathrm{d} v
$$

and to apply the classical formulae for the spontaneous emission : as our detection set up does not resolve the spectral shape of the fluorescence lines and as the detection is perpendicular to the laser beam, it is not necessary to take into account the frequency correlations between the laser and the fluorescence light [7], [8].

We shall express the density matrix as a perturbation expansion up to fourth order in the laser electric field. This method is very adequate to give a good understanding of physical processes and to allow a solution of the equation for any number of modes and for a sophisticated relaxation model, but it cannot give the exact shape of resonances for any laser intensity. In fact it is demonstrated that the fourth order is not sufficient in many experimental cases for which a method without perturbation is needed : this kind of calculation will be published later [10].

As it will appear that the relaxation processes have a major influence on the results, we shall take into account very carefully these processes (disorienting collisions, velocity diffusion by collisions and by trapping of fluorescence lines...). Nevertheless it will be necessary to make some crude approximations such as isotropy of relaxations and the use of the strong collisions model [11], [12] for velocity diffusion.

II. Equations of motion. - We assume the gas excitation by the discharge to be spatially homogeneous and the laser beam to be a plane wave. Therefore the density matrix depends only on the projection on the laser axis of the position $(r)$ and of the atomic velocity $(v)$. This approximation means that the 
length covered by any excited atom during its life time is negligible compared to the beam diameter. Among the several possible formulations for the equation giving $\rho(v, r, t)$, we shall choose the Boltzmann like formulation [11]:

$$
\begin{aligned}
& \dot{\rho}=\left(\frac{\partial}{\partial t}+v \frac{\partial}{\partial r}\right) \rho(v,, t)=-i[\mathcal{H}(r, t), \rho(v, r, t)]+ \\
&+\Lambda(v)+\left(\frac{\mathrm{d}}{\mathrm{d} t} \rho(v, r, t)\right)_{\text {relax }}
\end{aligned}
$$

where $\Lambda$ expresses the excitation by the discharge, $\mathcal{H}$ is the Hamiltonian and $(\mathrm{d} \rho / \mathrm{d} t)_{\text {relax }}$ contains all relaxation phenomena. Let us now study all terms of this equation and define some approximations. (For $\rho$, $\mathcal{H}$ and $\Lambda$ the formalism is the same as in pre vious publication [13] and will be only summarized.)

1) The DENSity MATRIX is restricted to the two levels connected to the laser transition ( $a$ is the lower level, $b$ the upper one). It is composed of four submatrices :

$$
\rho=\left(\begin{array}{cc}
{ }_{a} \rho & { }_{a b} \rho \\
{ }_{b a} \rho & { }_{b} \rho
\end{array}\right) .
$$

The non diagonal submatrices ${ }_{a b} \rho$ and ${ }_{b a} \rho$ represent optical macroscopic quantities, according to CohenTannoudji [14] we call them " optical coherences". $\rho$ and ${ }_{b} \rho$ represent the state of atoms in $a$ and $b$ levels : with the standard basis $|\mathrm{JM}\rangle$ their diagonal elements are the "populations " of the Zeeman sublevels and their off-diagonal elements are the « Zeeman coherences ".

Due to the symmetry of relaxation processes, we shall represent the density operator on the basis of normalized irreducible tensors [13], [15], [16], [17]

${ }_{\alpha \beta} \rho=\sum_{k Q}{ }_{\alpha \beta} \rho_{Q \alpha \beta}^{k} T_{Q}^{k} ; \quad{ }_{\alpha \beta} \rho_{Q}^{k}=\operatorname{Tr}\left(\rho_{{ }_{\alpha \beta}} T_{Q}^{k^{\dagger}}\right) ;$

$$
(\alpha=a \text { or } b) \text {. }
$$

As it is well known, ${ }_{\alpha} \rho_{0}^{0}$ defines the total population of the level $\alpha\left(n_{\alpha}=\sqrt{2 J_{\alpha}+1}{ }_{\alpha} \rho_{0}^{0}\right)$, the ${ }_{\alpha} \rho_{Q}^{1}$ define the three components of its orientation (magnetic dipole) and the ${ }_{\alpha} \rho_{Q}^{2}$ the five components of its alignment (electric quadrupole). ${ }_{\alpha} \rho_{0}^{k}$ components are called longitudinal quantities and determine the " populations " of the Zeeman sublevels. ${ }_{\alpha} \rho_{Q}^{k}$, with $Q \neq 0$, are transverse quantities related to the « Zeeman Coherences $»$. The three operators ${ }_{a b} \rho_{Q}^{1}$ are proportional to the optical electric dipole.

2) The excitation matrix $\Lambda(v)$ is assumed to be isotropic and homogeneous and to be proportional to a Maxwellian velocity distribution :

$$
\begin{aligned}
\Lambda(v)=W_{M}(v)\left[\lambda_{a} a\right. & \left.T_{0}^{0}+\lambda_{b}{ }_{b} T_{0}^{0}\right], \\
& W_{M}(v)=(u \sqrt{\pi})^{-1} \exp \left(-\frac{v^{2}}{u^{2}}\right) .
\end{aligned}
$$

The isotropy of $\Lambda$ is not rigourous in all experimental cases, as the trapping of some fluorescence lines can introduce alignment along the axes of the capillary cell [18]. This question is out of the scope of this paper (cf. ref. [4]).

3) The Hamiltonian is expressed as the sum of the single atom part $\left(\mathscr{H}_{0}\right)$, the Zeeman part $\left(\mathcal{H}_{\mathrm{z}}\right)$ and of the Hamiltonian of interaction with the laser beam $(R(r, t))$ :

$$
\begin{aligned}
\mathcal{H} & =\mathscr{H}+\mathcal{H}_{Z}+R(r, t) \\
\mathcal{H}= & W_{a} \sqrt{2 J_{a}+1_{a}} T_{0}^{0}+W_{b} \sqrt{2 J_{b}+1_{b}} T_{0}^{0} \\
\mathscr{H}_{Z}=-M_{z} H & =\omega_{a} \sqrt{\frac{J_{a}\left(J_{a}+1\right)\left(2 J_{a}+1\right)}{3}} T_{a}^{1}+ \\
& +\omega_{b} \sqrt{\frac{J_{b}\left(J_{b}+1\right)\left(2 J_{b}+1\right)}{3}}{ }_{b} T_{0}^{1} \\
R(r, t) & =-\mathbf{P} \cdot \mathbf{E}(r, t)=-\sum_{q}(-)^{q} P_{q} E_{-q}
\end{aligned}
$$

where $W_{a}$ and $W_{b}$ are the unperturbed level energies, $\omega_{a}$ and $\omega_{b}$ are the Zeeman splittings $\left(\omega_{\alpha}=g_{\alpha} \beta H\right.$; $g_{\alpha}=$ Landé factor ; $\beta=$ Bohr magneton). $\mathbf{P}$ is the electric dipole operator and $\mathbf{E}$ the laser beam electric field. $P_{q}$ and $E_{q}$ are the standard components:

$$
E_{0}=E_{z}, \quad E_{ \pm 1}=\frac{\mp 1}{\sqrt{2}}\left(E_{x} \pm i E_{y}\right)
$$

$\mathbf{P}$ has only off-diagonal optical matrix elements and can be expressed in the form $\left(P_{a b}=(-)^{J_{b}-J_{a}} P_{b a}^{*}\right.$ is the reduced matrix element) :

$$
P_{q}=\left(\frac{P_{a b}}{\sqrt{3}}\right)_{a b} T_{q}^{1}+\left(\frac{P_{b a}}{\sqrt{3}}\right)_{b a} T_{q}^{1} .
$$

The laser is composed of several modes

$$
\begin{aligned}
\mathbf{E}(r, t)=\sum_{\mu}\left[\boldsymbol{\delta}^{\mu} \exp \left[-i\left(\omega_{\mu} t-k_{\mu} r\right)\right]+\right. \\
\left.+\boldsymbol{\delta}^{\mu} \exp \left[i\left(\omega_{\mu} t-k_{\mu} r\right)\right]\right] .
\end{aligned}
$$

The complex vector $\boldsymbol{\delta}^{\mu}$ contains the relative phase of modes, but also all informations about the polarization (relative phases of the three components). As all modes have the same polarization defined by the complex vector e we shall use the notation :

$$
\boldsymbol{\delta}^{\mu}=\mathcal{E}^{\mu} \mathbf{e}=\sqrt{I_{\mu}} \exp \left(i \varphi_{\mu}\right) \mathbf{e} .
$$

Up to the paragraph VI we consider a travelling wave laser beam, therefore $k_{\mu}$ is positive. In our experiment the laser beam is parallel to the magnetic field $\left(k_{\mu}\right.$ parallel to $\left.O z\right)$, therefore $\varepsilon_{0}^{\mu}=0$.

4) Relaxation. - The relaxation phenomena include collisions and radiative effects. In ordinary optical pumping of a gas, with a broad line light 
source and in isotropic surroundings, the spherical symmetry of the relaxation allows us to write

$$
\left(\frac{\mathrm{d}_{\alpha \beta} \bar{\rho}_{Q}^{k}}{\mathrm{~d} t}\right)_{\text {coll }}=-\left(\Gamma_{\alpha \beta}(k)\right)_{\mathrm{coll} \alpha \beta} \bar{\rho}_{Q}
$$

for collisional relaxation, and

$$
\left(\frac{\mathrm{d}{ }_{\beta} \bar{\rho}_{Q}^{k}}{\mathrm{~d} t}\right)_{\mathrm{rad}}=\left[-\gamma_{\beta}+\sum_{i} \gamma_{\beta i} \alpha_{\beta}(k, i) x_{i}\right]_{\beta} \bar{\rho}_{Q}^{k}
$$

for the radiative relaxation in presence of trapping [19], [20] of the fluorescence lines $\beta \rightarrow i$. Where $\gamma_{\beta}=1 / \tau_{\beta}$ is the sum of all the transition probabilities $\gamma_{\beta i}, \alpha_{\beta}(k, i)$ is a coefficient tabulated [21] as a function of $k, J_{\beta}$ and $J_{i}$, and $x_{i}$ is the averaged probability for a photon $\beta \rightarrow i$ to be reabsorbed before leaving the cell. Eq. (12) and (13) must be applied to the total density matrix $\left(\bar{\rho}=\int \rho(v) \mathrm{d} v\right)$ and they are valid only if the velocity distribution of pumped atoms is isotropic (namely $\rho(v)=\bar{\rho} W_{\mathrm{M}}(v)$ ). These two conditions are not fullfilled by the optical pumping with a laser beam : due to coherence and monochromaticity eq. (2) must be written for $\rho(v)$ which is not proportional to $W_{\mathrm{M}}(v)$ (hole burning). For a given velocity, the relaxation has only the cylindrical symmetry and it introduces a coupling between the different tensorial orders (the component index $Q$ being conserved [22]). Furthermore we must take into account the velocity changes produced by collisions and by trapping of the fluorescence lines. As far as the Boltzmann equation (with classical description of the atomic motion) can be used, the most general form of the collisional relaxation term can be shown to be :

$$
\begin{gathered}
\left(\frac{\mathrm{d}}{\mathrm{d} t}{ }_{\alpha \beta} \rho_{Q}^{k}(v, r, t)\right)_{\text {coll }}=\sum_{k^{\prime}}\left[-{ }_{\alpha \beta} B_{Q}^{k k^{\prime}}(v)_{\alpha \beta} \rho_{Q}^{k^{\prime}}(v, r, t)+\right. \\
\left.+\int_{\alpha \beta} A_{Q}^{k k^{\prime}}\left(v, v^{\prime}\right)_{\alpha \beta} \rho_{Q}^{k^{\prime}}\left(v^{\prime}, r, t\right) \mathrm{d} v^{\prime}\right] .
\end{gathered}
$$

To avoid inextricable complications we must simplify this expression with some crude approximations [23] : 1) We assume that for each velocity the relaxation is isotropic enough to produce no coupling between the different tensorial orders and to give rise to relaxation rates independent of $Q$. This approximation is valid for light perturbers $(\mathrm{He})$ for which the relative velocity is very close to the perturber velocity and therefore isotropic. 2) Like many authors [11], [12], we use the strong collision model for velocity changes (no memory of the initial speed). Contrary to the previous one, this approximation is better with heavy perturbers which affect more strongly the velocity of studied atoms [24].

The trapping of fluorescence lines produces a restitution of atomic quantities, the form of which is comparable to the second term of (14). A further coupling between different positions can be ignored as it will be shown that $\rho(v, r, t)$ varies but slowly with $r$. We simplify this term with the same approximation as for collision (isotropy, no speed memory) so that the total relaxation terms become :

$$
\begin{aligned}
\left(\frac{\mathrm{d}}{\mathrm{d} t}{ }_{\beta} \rho_{Q}^{k}(v, r, t)\right)_{\mathrm{rel}} & =-\Gamma_{\beta}^{\prime}(k)_{\beta} \rho_{Q}^{k}(v, r, t)+ \\
& +\gamma_{\beta}^{\prime}(k) W_{\mathrm{M}}(v) \int{ }_{\beta} \rho_{Q}^{k}\left(v^{\prime}, r, t\right) \mathrm{d} v^{\prime}
\end{aligned}
$$

$$
\begin{array}{r}
\left(\frac{\mathrm{d}}{\mathrm{d} t}{ }_{a b} \rho_{Q}^{k}(v, r, t)\right)_{\text {rel }}=-G_{a b}(k)_{a b} \rho_{Q}^{k}(v, r, t)+ \\
+g_{a b}(k) W_{\mathrm{M}}(v) \int{ }_{a b} \rho_{Q}^{k}\left(v^{\prime}, r, t\right) \mathrm{d} v^{\prime}
\end{array}
$$

In each of these expressions the first term is the rate of decrease of the studied quantity, either by destruction, or by velocity change of the atom. The second term expresses the arrival from any other velocity $v^{\prime}$ of atoms bearing the quantity ${ }_{\alpha \beta} T_{Q}^{k}$. For the atomic quantities (eq. (15)) the relaxation rates are real (impact approximation and spherical symmetry) and given by :

$$
\begin{aligned}
& \Gamma_{\beta}^{\prime}(k)=\gamma_{\beta}+\left(\Gamma_{\beta}^{\prime}(k)\right)_{\mathrm{coll}}, \quad \gamma_{\beta}=\frac{1}{\tau_{\beta}} \\
& \gamma_{\beta}^{\prime}(k)=\sum_{i} \gamma_{\beta i} \alpha_{\beta}(k, i) x_{i}+\left(\gamma_{\beta}^{\prime}(k)\right)_{\mathrm{coll}}
\end{aligned}
$$

with these approximations, the velocity changing collisions and the trapping of fluorescence lines appear to have the same effect for velocity thermalisation of atomic quantities. By integration over $v$, (15) gives again the classical relaxation formula (12) + (13) with the " classical relaxation rate ":

$$
\Gamma_{\beta}(k)=\Gamma_{\beta}^{\prime}(k)-\gamma_{\beta}^{\prime}(k) .
$$

It describes the relaxation of the quantity $T_{Q}^{k}$ without any regard to the atomic velocity. It is the relaxation rate which occurs in optical pumping with ordinary light sources [23].

In (16) the relaxation rates of optical quantities can be complex to take into account the frequency shift produced by collisions :

$$
\begin{array}{r}
G_{a b}(k)=G_{b a}^{*}(k)=\frac{1}{2}\left(\gamma_{a}+\gamma_{b}\right)+\left(G_{a b}(k)\right)_{\mathrm{coll}}= \\
=\Gamma_{a b}^{\prime}(k)-i \Delta(k)
\end{array}
$$

$$
g_{a b}(k)=g_{b a}^{*}(k)=\gamma_{a b}^{\prime}(k)-i \Delta^{\prime}(k)
$$

the arrival rate $g_{a b}(k)$ is due only to collisions. Its imaginary part takes into account the correlations between phase shift and velocity changes, according to Rautian's theory [11].

Finally we must take into account the transfer of atomic quantities by spontaneous emission from 
the upper level $b$ to the lower level $a$. According to Ducloy [25], we write :

$$
\begin{aligned}
& \left(\frac{\mathrm{d}}{\mathrm{d} t}{ }_{a} \rho_{Q}^{k}\right)_{\mathrm{tr}}=\Theta(b, a, k)_{b} \rho_{Q}^{k}=- \\
& -(-)^{J_{a}+J_{b}+k} \gamma_{b a}\left(2 J_{b}+1\right)\left\{\begin{array}{ccc}
k & J_{b} & J_{b} \\
1 & J_{a} & J_{a}
\end{array}\right\}_{b} \rho_{Q}^{k} .
\end{aligned}
$$

III. Calculation of the density matrix $\left(J_{a}=0\right.$, $J_{b}=1$ case). - The calculation in this paragraph follows a well known scheme. In a different form, it is completely equivalent to the Lamb's method [9] (iteration, rotating wave approximation, Doppler approximation, neglecting of the difference of the Doppler effect between two modes...). The difference comes from the use of irreducible tensors to take into account the Zeeman structure and the relaxations.
1) Perturbation method. - This method consists in developing eq. (2) into an iterative set of equations :

$$
\begin{aligned}
{ }^{(0)} \dot{\rho}=-i\left[\mathcal{H}_{0}+\mathcal{H}_{\mathrm{Z}},{ }^{(0)} \rho\right]+\left(\frac{\mathrm{d}^{(0)} \rho}{\mathrm{d} t}\right)_{\mathrm{rel}}+\Lambda \\
{ }^{(n)} \dot{\rho}=-i\left[\mathcal{H}_{0}+\mathcal{H}_{\mathrm{Z}},{ }^{(n)} \rho\right]+ \\
+\left(\frac{\mathrm{d}^{(n)} \rho}{\mathrm{d} t}\right)_{\mathrm{rel}}-i\left[R,{ }^{(n-1)} \rho\right] .
\end{aligned}
$$

The components of the density matrix on the basis of irreducible tensors are obtained by projecting eq. (21) on this basis according to (4). $\Lambda$ being scalar and $R$ containing only optical off-diagonal elements, it is clear that even orders contain only atomic quantities and odd orders optical coherences. After some algebra [4], eq. (21) become :

$$
\begin{aligned}
& { }_{a}^{(2 n)} \dot{\rho}_{Q}^{k}(v)=-\left[i Q \omega_{a}+\Gamma_{a}^{\prime}(k)\right]^{(2 n)} \rho_{Q}^{k}(v)+\gamma_{a}^{\prime}(k) W_{\mathrm{M}}(v){ }_{a}^{(2 n)} \rho_{Q}^{k}+\Theta(b, a, k){ }_{b}^{(2 n)} \rho_{Q}^{k}(v) \\
& +i \sum_{k^{\prime} Q^{\prime} q}(-)^{k+k^{\prime}} \underset{a b}{a} G_{Q^{\prime} Q}^{k^{\prime} k} E_{-q}\left[{ }_{a b}^{(2 n-1)} \rho_{Q^{\prime}}^{k^{\prime}}(v) P_{a b}^{*}+(-)^{k+k^{\prime}+Q^{\prime}(2 n-1)} \underset{a b}{\left(2 n Q^{\prime}\right.}(v) P_{a b}\right] \\
& { }_{b}^{(2 n)} \dot{\rho}_{Q}^{k}(v)=-\left[i Q \omega_{b}+\Gamma_{b}^{\prime}(k)\right]_{b}^{(2 n)} \rho_{Q}^{k}(v)+\gamma_{b}^{\prime}(k) W_{\mathrm{M}}(v){ }_{b}^{(2 n)} \rho_{Q}^{k} \\
& +i \sum_{k^{\prime} Q^{\prime} q}{ }_{b a}^{q} G_{Q^{\prime} Q}^{k^{\prime} k} E_{-q}\left[\begin{array}{c}
(2 n-1) \\
a b
\end{array} \rho_{Q^{\prime}}^{k^{\prime}}(v) P_{a b}^{*}+(-)^{k+k^{\prime}+Q^{\prime}(2 n-1)}{ }_{a b}^{k_{Q^{\prime}}^{\prime *}}(v) P_{a b}\right] \\
& \underset{a b}{(2 n+1)} \dot{\rho}_{Q^{\prime}}^{k^{\prime}}=-\left[i\left(\frac{\omega_{a}+\omega_{b}}{2} Q^{\prime}-\omega\right)+G_{a b}(k)\right]{ }_{a b}^{(2 n+1)} \rho_{Q^{\prime}}^{k^{\prime}}(v)+g_{a b}(k) W_{M}(v){ }_{a b}^{(2 n+1)} \rho_{Q^{\prime}}^{k^{\prime}} \\
& -i \frac{\omega_{a}-\omega_{b}}{2}\left\{Q^{\prime} \frac{J_{a}\left(J_{a}+1\right)-J_{b}\left(J_{b}+1\right)}{k^{\prime}\left(k^{\prime}+1\right)}{ }_{(2 n+1)}^{\left({ }_{a b}\right.} \rho_{Q^{\prime}}^{k^{\prime}}(v)+a(k){ }_{a b}^{(2 n+1)} \rho_{Q^{\prime}}^{k^{\prime}-1}(v)+\right. \\
& \left.+a(k+1)^{(2 n+1)} \rho_{a b}^{k_{Q^{\prime}}+1}(v)\right\} \\
& \left.+i \sum_{k Q q}(-)^{q} E_{q\left[b_{a}\right.} G_{Q^{\prime} Q}^{k^{\prime} k}{ }_{b}^{(2 n)} \rho_{Q}^{k}(v)+(-)^{k+k^{\prime}} \underset{a b}{q} G_{Q^{\prime} Q}^{k^{\prime} k}{ }_{a}^{(2 n)} \rho_{Q}^{k}(v)\right]
\end{aligned}
$$

where $\omega=W_{b}-W_{a}$ is the atomic frequency and where the geometrical factors are defined by:

$$
\begin{aligned}
{ }_{\alpha \beta}^{q} G_{Q^{\prime} Q}^{k^{\prime} k} & =(-)^{J_{\alpha}+J_{\beta}+Q^{\prime}} \sqrt{(2 k+1)\left(2 k^{\prime}+1\right)}\left(\begin{array}{ccc}
k^{\prime} & 1 & k \\
Q^{\prime} & q & -Q
\end{array}\right)\left\{\begin{array}{ccc}
k^{\prime} & 1 & k \\
J_{\alpha} & J_{\alpha} & J_{\beta}
\end{array}\right\} \\
a(k) & =k^{-1}\left\{\left[\left(J_{a}+J_{b}+1\right)^{2}-k^{2}\right]\left[k^{2}-\left(J_{a}-J_{b}\right)^{2}\right]\left[k^{2}-Q^{2}\right]\left[4 k^{2}-1\right]^{-1}\right\}^{1 / 2} .
\end{aligned}
$$

When the $(2 n-1)$ order has been calculated the eq. $(22 b)$ are easy to solve since they do not couple different tensorial orders. The solutions of $(22 b)$ are then inserted in the transfer term of eq. (22a) which are equally easy to solve. On the other hand in eq. (22c) the Zeeman term introduces a coupling between the different tensorial orders. This is due to the fact that ${ }_{a b} \rho_{Q}^{k}$ has no characteristic frequency, as it is a linear superposition of all the $\rho_{M_{a} M_{b}}$ matrix elements with $M_{a}-M_{b}=Q$, which correspond to different optical frequencies. There are two simple cases for which the coupling disappears : first, when the Landé factor of the two levels are equal $\left(\omega_{a}=\omega_{b}\right)$. Secondly when $J=0$ for one of the laser levels (only $k=1$ is possible for optical coherences). To avoid complication, in the following discussion, we shall restrict ourself to the case $J_{a}=0, J_{b}=1$. The general case is studied in reference [4] and some results will be summarized in paragraph VII.

2) GeNeral Form of the Stationary SOlution. At all orders of perturbation, the equations have the general form :

$$
\begin{aligned}
& \left(\frac{\partial}{\partial t}+v \frac{\partial}{\partial r}\right) f(v, r, t)= \\
& =\left(i \Omega-\Gamma^{\prime}\right) f(v, r, t)+\gamma^{\prime} W_{\mathrm{M}}(v) \int f\left(v^{\prime}, r, t\right) \mathrm{d} v^{\prime}+ \\
& \quad+\sum_{\Omega_{n}} A\left(v, \Omega_{n}\right) \exp \left[i\left(\Omega_{n} t-K_{n} r\right)\right]
\end{aligned}
$$


where $\Omega$ is the resonance frequency of the quantity $f$ and $\Omega_{n}$ are the different frequencies which appear at the $n$th order. The stationary solution has the general form :

$$
f(v, r, t)=\sum_{\Omega_{n}} f\left(v, \Omega_{n}\right) \exp \left(i\left[\Omega_{n} t-K_{n} r\right]\right) .
$$

Eq. (25), solved separately for each frequency, gives :

$$
\begin{array}{r}
f\left(v, \Omega_{n}\right)=\left[A\left(v, \Omega_{n}\right)+\gamma^{\prime} W_{\mathrm{M}}(v) \int f\left(v^{\prime}, \Omega_{n}\right) \mathrm{d} v^{\prime}\right] \times \\
\times\left[\Gamma^{\prime}-i\left(\Omega-\Omega_{n}+K_{n} v\right)\right]^{-1} .
\end{array}
$$

The physical meaning of this expression is clear: the first term, $A\left(v, \Omega_{n}\right) /\left[\Gamma^{\prime}-i\left(\Omega-\Omega_{n}+K_{n} v\right)\right]$, represents atoms whose speed has not been changed. It is the ratio of the excitation rate (for the concerned $n$th order), $A\left(v, \Omega_{n}\right)$, to a resonant denominator which depends on the total relaxation rate $\Gamma^{\prime}$, including probability of velocity changes. The second term represents atoms whose speed has changed once or more. It is proportional to $\gamma^{\prime}$ which is the rate of velocity changes without destruction of the quantity $f$.
Integrating (27) over $v$ one obtains :

$$
\begin{aligned}
\int f\left(v, \Omega_{n}\right) \mathrm{d} v & =\int \frac{A\left(v, \Omega_{n}\right) \mathrm{d} v}{\Gamma^{\prime}-i\left(\Omega-\Omega_{n}+K_{n} v\right)} \times \\
\times\left[1-\gamma^{\prime} \int \frac{W_{M}(v) \mathrm{d} v}{\Gamma^{\prime}-i\left(\Omega-\Omega_{n}+K_{n} v\right)}\right]^{-1} & .
\end{aligned}
$$

Inserting (28) into (27) one finally gets the general form for $f\left(v, \Omega_{n}\right)$.

Approximations. - At each order of perturbation, we shall perform the rotating wave approximation: we shall neglect off resonance terms for which $\Omega-\Omega_{n}$ is of the order of magnitude of the optical frequency $\omega$. Therefore, only positive frequencies, of the order of $\omega$, are to be kept for optical coherences ${ }_{a b} \rho$ ( $-\omega$ for ${ }_{b a} \rho$ ), and low frequencies, of the order of beat notes $\omega_{v}-\omega_{\mu}$, for atomic quantities ${ }_{\alpha} \rho$.

At all even orders (atomic quantities), $K_{2 n} v$ is of the order of $\left(k_{v}-k_{\mu}\right) v$. As we are concerned with a travelling wave, $k_{v}$ and $k_{\mu}$ are positive. Therefore $K_{2 n} v$ can be neglected in (27) and (28): $\left(k_{v}-k_{\mu}\right) v \sim\left(k_{v}-k_{\mu}\right) u \sim$ $\sim 10^{-7} k_{v} u \sim 100 \mathrm{~Hz}$, for a $2 m$ laser (27) and (28) becomes $\left(\Gamma=\Gamma^{\prime}-\gamma^{\prime}\right)$ :

$$
\begin{aligned}
\bar{f}\left(\Omega_{2 n}\right) & =\int f\left(v, \Omega_{2 n}\right) \mathrm{d} v=\frac{\int A\left(v, \Omega_{2 n}\right) \mathrm{d} v}{\Gamma-i\left(\Omega-\Omega_{2 n}\right)} \\
f\left(v, \Omega_{2 n}\right) & =\frac{1}{\Gamma^{\prime}-i\left(\Omega-\Omega_{2 n}\right)}\left[A\left(v, \Omega_{2 n}\right)+\frac{\gamma^{\prime} W_{\mathrm{M}}(v)}{\Gamma-i\left(\Omega-\Omega_{2 n}\right)} \int A\left(v, \Omega_{2 n}\right) \mathrm{d} v\right] \\
& =\frac{A\left(v, \Omega_{2 n}\right)}{\Gamma^{\prime}-i\left(\Omega-\Omega_{2 n}\right)}+\left[\frac{1}{\Gamma-i\left(\Omega-\Omega_{2 n}\right)}-\frac{1}{\Gamma^{\prime}-i\left(\Omega-\Omega_{2 n}\right)}\right] W_{\mathrm{M}}(v) \int A\left(v, \Omega_{2 n}\right) \mathrm{d} v .
\end{aligned}
$$

3) RESUltS AT EVEN ORDERS. - The algebric development, up to fourth order, is very cumbersome to write [4], therefore we shall only give the results for even orders in the simple case $J_{a}=0 ; J_{b}=1$. Odd orders would provide the macroscopic optical polarization of the medium, useful for the laser theory, but this is beyond the scope of this paper.

At zero order the source term in (22a) and (22b) comes from $\Lambda(v)=W_{\mathrm{M}}(v)\left[\lambda_{a} a T_{0}^{0}+\lambda_{b} T_{0}^{0}\right]$. There- fore the only non vanishing terms are the populations. With $\alpha=a$ or $b$, (29) and (30) give

$$
{ }_{\alpha}^{(0)} \rho_{0}^{0}(v)=W_{\mathrm{M}}(v){ }_{\alpha}^{(0)} \bar{\rho}_{0}^{0}=\frac{\lambda_{\alpha}}{\Gamma_{\alpha}(0)} W_{\mathrm{M}}(v) \text {. }
$$

This expression depends only on the classical relaxation rate $\Gamma_{\alpha}(0)=\Gamma_{\alpha}^{\prime}(0)-\gamma_{\alpha}^{\prime}(0)$. Indeed at zero order velocity diffusion is not observable since this process does not affect the Maxwellian distribution introduced by $\Lambda(v)$.

At second order, we obtain :

$$
\begin{gathered}
{ }_{b}^{(2)} \rho_{Q}^{k}(v)=n \frac{\left|P_{a b}\right|^{2}}{3} \sqrt{2 k+1} \sum_{\substack{v \mu \\
q_{1} q_{2}}}(-)^{q+k}\left(\begin{array}{ccc}
1 & 1 & k \\
q_{1} & q_{2} & -Q
\end{array}\right) b_{Q}^{k}(v, v-\mu) \varepsilon_{q_{1}}^{v^{*}} \varepsilon_{-q_{2}}^{\mu} \times \\
\quad \times \exp \left\{i\left[\left(\omega_{v}-\omega_{\mu}\right) t-\left(k_{v}-k_{\mu}\right) r\right]\right\} \\
{ }_{a}^{(2)} \rho_{0}^{0}(v)=n \frac{\left|P_{a b}\right|^{2}}{3} \sum_{v \mu q_{1}} a(v, v-\mu) \mathcal{E}_{q_{1}}^{v^{*}} \delta_{q_{1}}^{\mu} \exp \left\{i\left[\left(\omega_{v}-\omega_{\mu}\right) t-\left(k_{v}-k_{\mu}\right) r\right]\right\}
\end{gathered}
$$


where $n=\lambda_{b} /\left(\Gamma_{b}(0) \sqrt{3}\right)-\lambda_{a} / \Gamma_{a}$, is the population inversion. Using (30) we have :

$$
\begin{gathered}
b_{Q}^{k}(v, v-\mu)=\frac{A_{q_{1} q_{2}}^{v \mu}(v)}{\Gamma_{b}^{\prime}(k)+i\left(\omega_{v}-\omega_{\mu}+Q \omega_{b}\right)}+\left[\frac{1}{\Gamma_{b}(k)+i\left(\omega_{v}-\omega_{\mu}+Q \omega_{b}\right)}-\frac{1}{\Gamma_{b}^{\prime}(k)+i\left(\omega_{v}-\omega_{\mu}+Q \omega_{b}\right)}\right] \times \\
\times W_{\mathrm{M}}(v) \int A_{q_{1} q_{2}}^{v \mu}\left(v^{\prime}\right) \mathrm{d} v^{\prime} \\
a(v, v-\mu)=\frac{A_{q_{1}-q_{1}}^{v \mu}(v)}{\left[\Gamma_{a}^{\prime}+i\left(\omega_{v}-\omega_{\mu}\right)\right]_{t}}+\left\{\frac{1}{\left[\Gamma_{a}+i\left(\omega_{v}-\omega_{\mu}\right)\right]_{t}}-\frac{1}{\left[\Gamma_{a}^{\prime}+i\left(\omega_{v}-\omega_{\mu}\right)\right]_{t}}\right\} W_{\mathrm{M}}(v) \int A_{q_{1}-q_{1}}^{v \mu}\left(v^{\prime}\right) \mathrm{d} v^{\prime}
\end{gathered}
$$

where the subscript $t$ means corrected values taking into account the transfer by spontaneous emission according to

$$
\begin{aligned}
{\left[\Gamma_{a}^{(\prime)}+\right.} & \left.i\left(\omega_{v}-\omega_{\mu}\right)\right]_{t}^{-1}=\left[\Gamma_{a}^{(\prime)}+i\left(\omega_{v}-\omega_{\mu}\right)\right]^{-1} \times \\
& \times\left\{1-\gamma_{b a}\left[\Gamma_{b}^{(\prime)}(0)+i\left(\omega_{v}-\omega_{\mu}\right)\right]^{-1}\right\} .
\end{aligned}
$$

In (34) and (35), the first term is the contribution of atoms whose velocity has not changed; the second one expresses the survival of a part of atomic quantities during velocity diffusion processes. The source term, from the first order is given by :

$$
\begin{aligned}
& A_{q_{1} q_{2}}^{v \mu}(v)= \\
= & W_{M}(v)\left\{\frac{\left[1-g_{a b} W\left(\omega-q_{1} \omega_{b}-\omega_{v}+i \Gamma_{a b}^{\prime}\right)\right]^{-1}}{\Gamma_{a b}^{\prime}-i\left(\omega-q_{1} \omega_{b}-\omega_{v}+k_{v} v\right)}+\right. \\
+ & \left.\frac{\left[1-g_{a b}^{*} W^{*}\left(\omega+q_{2} \omega_{b}-\omega_{\mu}+i \Gamma_{a b}^{\prime}\right)\right]^{-1}}{\Gamma_{a b}^{\prime}+i\left(\omega+q_{2} \omega_{b}-\omega_{\mu}+k_{\mu} v\right)}\right\} \cdot \quad
\end{aligned}
$$

The denominators express the optical resonance of mode $v$ (or $\mu$ ) and the factors at the form

$$
\left[1-g_{a b} W\left(\omega-q_{1} \omega_{b}-\omega_{v}+i \Gamma_{a b}^{\prime}\right)\right]^{-1}
$$

express the partial conservation of optical quantities (at first order) during velocity changes. In these factors $W$ is defined by

$$
\begin{gathered}
W(\Omega+i \Gamma)=\int_{-\infty}^{+\infty} \frac{W_{\mathrm{M}}(v) \mathrm{d} v}{\Gamma-i(\Omega+\mathrm{k} v)}= \\
=\frac{1}{u \sqrt{\pi}} \int_{-\infty}^{\infty} \frac{\exp \left(-v^{2} / u^{2}\right) \mathrm{d} v}{\Gamma-i(\Omega+\mathrm{k} v)} \\
=-\frac{i}{\Delta v} Z\left(\frac{\Omega}{\Delta v}+i \frac{\Gamma}{\Delta v}\right)= \\
=\frac{1}{\Delta v}[X(\Omega)+i Y(\Omega)]
\end{gathered}
$$

where $Z$ is the plasma dispersion function [26] and $\Delta v=\mathrm{k} u$ is the Doppler width.

In (37) and in all the following discussion, the imaginary part of $G_{a b}$ has been included in $\omega$ which is now the atomic optical frequency shifted by colli- sions. Integrating $A_{q_{1} q_{2}}^{v \mu}(v)$ over $v$ with the help of (38), we find :

$$
\begin{gathered}
\int A_{q_{1} q_{2}}^{v \mu}(v)=\frac{W\left(\omega-q_{1} \omega_{b}-\omega_{v}+i \Gamma_{a b}^{\prime}\right)}{1-g_{a b} W\left(\omega-q_{1} \omega_{b}-\omega_{v}+i \Gamma_{a b}^{\prime}\right)}+ \\
+\frac{W^{*}\left(\omega+q_{2} \omega_{b}-\omega_{\mu}+i \Gamma_{a b}^{\prime}\right)}{1-g_{a b}^{*} W^{*}\left(\omega+q_{2} \omega_{b}-\omega_{\mu}+i \Gamma_{a b}^{\prime}\right)} .
\end{gathered}
$$

In zero magnetic field, this is exactly the optical line shape obtained by Rautian [11].

To obtain the expression of the fluorescent light we need the total density matrix

$$
\begin{aligned}
& \underset{b}{(2) \rho_{Q}^{k}}=n \frac{\left|P_{a b}\right|^{2}}{3} \sqrt{2 k+1} \times \\
& \times \sum_{\substack{v \mu \\
q_{1} q_{2}}}(-)^{k+q_{1}}\left(\begin{array}{ccc}
1 & 1 & k \\
q_{1} & q_{2} & -Q
\end{array}\right) \mathcal{E}_{q_{1}}^{v_{1}^{*}} \varepsilon_{-q_{2}}^{\mu} \\
& \times \frac{\exp \left\{i\left[\left(\omega_{v}-\omega_{\mu}\right) t-\left(k_{v}-k_{\mu}\right) r\right]\right\} \int A_{q_{1} q_{2}}^{v \mu}(v) \mathrm{d} v}{\Gamma_{b}(k)+i\left(\omega_{v}-\omega_{\mu}+Q \omega_{b}\right)}
\end{aligned}
$$

and a similar expression for the a level. Eq. (40) allows us to calculate the modulations of the fluorescence lines which are resonant for $\omega_{v}-\omega_{\mu}=Q \omega_{b}$ and the Hanle effect which is provided by the unmodulated terms $(v=\mu)$. It is important to notice that (40) depends only on the classical relaxation rate $\Gamma_{b}(k)$ (cf. (18)). It differs from the results of the theory without velocity changes [13] by the rather insensitive optical factor $\int A_{q_{1} q_{2}}^{v \mu}(v) \mathrm{d} v$. In particular the shape of the Hanle effect is not modified, and the measurement of the Hanle effect width still yields the classical relaxation rate [27].

Fourth order gives the first non linear correction to the linear atomic response obtained at second order. ${ }^{(4)} \bar{\rho}_{+2}^{2}$ provides a correction to the Hanle effect shape (radiative broadening), but, as explained $\mathrm{n}$ the introduction, fourth order is quite insufficient to give the curve shape for any laser intensity [10]. 
Therefore we shall focus our interest only on longitudinal quantities, ${ }_{\alpha}^{(4)} \bar{\rho}_{0}^{k}$, which will give us (at least qualitatively) an interpretation of observed saturation phenomena. Furthermore, as we will not observe the modulations of fluorescence light, we keep only unmodulated terms for which the four interacting modes are such as $\omega_{v}-\omega_{\mu}+\omega_{\lambda}+\omega_{\kappa}=0$. For the present, we assume the modes to be equidistant with a frequency spacing $\Delta \omega$.

To avoid algebric complications, we shall write formulae only for the studied experimental case for which the laser beam is $\sigma$ linearly polarized.

$$
e_{0}=0 ;\left|e_{1}\right|=\left|e_{-1}\right|=\frac{1}{\sqrt{2}} .
$$

In that case there is no orientation (so long as the Zeeman splitting $\omega_{b}$ is small compared to the Doppler width $\Delta v$ ) and all other longitudinal quantities are proportional to each other (this is true only for $\left.J_{a}=0, J_{b}=1\right)$. We obtain the saturation signal :

$$
\begin{aligned}
S=- & \Gamma_{a}\left[1-\frac{\gamma_{b a}}{\Gamma_{b}(0)}\right]^{-1}{ }_{a}^{(4)} \rho_{0}^{0}=\sqrt{3} \Gamma_{b}(0) \underset{b}{(4)} \rho_{0}^{0}=\sqrt{6} \Gamma_{b}(2){ }_{b}^{(4)} \rho_{0}^{2} \\
= & \frac{n\left|P_{a b}\right|^{4}}{36} \sum_{\substack{v-\mu+\lambda-\kappa=0 \\
q_{4}, q_{1}= \pm 1}} \frac{\mathcal{E}^{v^{*}} \mathcal{E}^{\mu} \varepsilon^{k^{*}} \varepsilon^{\lambda}}{1-g_{a b} W\left(\omega+q_{4} \omega_{b}-\omega_{\kappa}+i \Gamma_{a b}^{\prime}\right)} \int \frac{\mathrm{d} v}{\Gamma_{a b}^{\prime}-i\left(\omega+q_{4} \omega_{b}-\omega_{\kappa}+k_{\kappa} v\right)} \times \\
& \times\left[a(v, v-\mu)+\sum_{\substack{k Q \\
q_{2}, q_{3}= \pm 1}}(2 k+1)\left(\begin{array}{ccc}
1 & 1 & k \\
q_{1} & q_{2} & -Q
\end{array}\right)\left(\begin{array}{ccc}
k & 1 & 1 \\
Q & q_{3} & q_{4}
\end{array}\right) b_{Q}^{k}(v, v-\mu)\right]+c c
\end{aligned}
$$

$a(v, v-\mu)$ and $b_{Q}^{k}(v, v-\mu)$ express the contribution of the second order atomic quantities modulated at frequency $\omega_{v}-\omega_{\mu}$ and demodulated by interaction with modes $\kappa$ and $\lambda\left(\omega_{v}-\omega_{\mu}=\omega_{\kappa}-\omega_{\lambda}\right)$. The denominator $\Gamma_{a b}^{\prime}-i\left(\omega+q_{4} \omega_{b}-\omega_{\kappa}+k_{\kappa} v\right)$ arises from third order; it is resonant when the Doppler shifted third order frequency,

$$
\omega_{v}-\omega_{\mu}+\omega_{\lambda}-\left(k_{v}-k_{\mu}+k_{\lambda}\right) v=\omega_{\kappa}-k_{\kappa} v,
$$

is equal to the atomic frequency $\omega+q_{4} \omega_{b}$. Finally the denominator $1-g_{a b} W($ ) arises from velocity changes at third order, exactly as it did at first order in (37). All possible combinations of the $q_{i}$ values,

\begin{tabular}{|c|c|c|c|c|c|c|c|c|c|c|}
\hline Case & $q_{1}$ & $q_{2}$ & $Q$ & $q_{3}$ & $q_{4}$ & $q_{2}-q_{4}$ & $\varepsilon_{q_{1}}^{\nu} \varepsilon_{-q_{2}}^{\mu}$ & ${ }^{(2)} \rho_{Q}^{k}(2 \mathrm{nd}$ order $)$ & $\varepsilon_{q_{3}}^{\lambda}$ & $\varepsilon_{-q_{4}}^{\kappa}$ \\
\hline$a(\mathrm{PE})$ & -1 & 1 & 0 & 1 & -1 & 2 & $\sigma^{+} \sigma^{+}$ & Longitudinal quantities, & $\sigma^{-}$ & $\sigma^{-}$ \\
\hline$b(\mathrm{PE})$ & 1 & -1 & 0 & 1 & -1 & 0 & $\left.\sigma^{-} \sigma^{-}\right\}$ & ${ }_{b}^{(2)} \rho_{0}^{k}$ and ${ }_{a}^{(2)} \rho_{0}^{0}$ & $\sigma^{-}$ & $\sigma^{-}$ \\
\hline$c(\mathrm{ZCE})$ & 1 & 1 & 2 & -1 & -1 & 2 & $\sigma^{-} \sigma^{+}$ & Transverse alignment in $b,{ }_{b}^{(2)} \rho_{2}^{2}$ & $\sigma^{+}$ & $\sigma^{-}$ \\
\hline
\end{tabular}
with their physical meaning, are shown in Table I.

$$
\begin{aligned}
\frac{1}{\Gamma_{b}^{\prime}(k)+i\left(\omega_{v}-\omega_{\mu}+Q \omega_{b}\right)} \int \frac{W_{\mathrm{M}}(v) \mathrm{d} v}{\Gamma_{a b}^{\prime}-i\left(\omega-\omega_{\kappa}+q_{4} \omega_{b}+\mathrm{k} v\right)} \times \\
\times\left[\frac{1}{\Gamma_{a b}^{\prime}-i\left(\omega-\omega_{v}-q_{1} \omega_{b}+\mathrm{k} v\right)}+\frac{1}{\Gamma_{a b}^{\prime}+i\left(\omega-\omega_{\mu}+q_{2} \omega_{b}+\mathrm{k} v\right)}\right]= \\
=\frac{1}{\Gamma_{b}^{\prime}(k)+i\left(\omega_{v}-\omega_{\mu}+Q \omega_{b}\right)}\left[\frac{W\left(\omega-\omega_{v}-q_{1} \omega_{b}+i \Gamma_{a b}^{\prime}\right)-W\left(\omega-\omega_{\kappa}+q_{4} \omega_{b}+i \Gamma_{a b}^{\prime}\right)}{i\left(\omega_{\kappa}-\omega_{v}-\left(q_{4}+q_{1}\right) \omega_{b}\right)}+\right. \\
\left.+\frac{W^{*}\left(\omega-\omega_{\mu}+q_{2} \omega_{b}+i \Gamma_{a b}^{\prime}\right)+W\left(\omega-\omega_{\kappa}+q_{4} \omega_{b}+i \Gamma_{a b}^{\prime}\right)}{2 \Gamma_{a b}+i\left(\omega_{\kappa}-\omega_{\mu}+\left(q_{2}-q_{4}\right) \omega_{b}\right)}\right] .
\end{aligned}
$$

Table I

To obtain all possible combinations one must, for each of the 3 lines, take into accound the symmetric cases where $\sigma^{+}$and $\sigma^{-}$ are exchanged and the signs of the $q_{i}$ and $Q$ are inverted. As the laser beam is linearly $\sigma$ polarized each mode has 2 components, $\sigma^{+}$ and $\sigma^{-}:$in all cases, we indicate which is used for each mode. 
In this expression we have neglected the differences between wave vectors and we have set

$$
k_{v} \simeq k_{\mu} \simeq k_{\kappa} \simeq k_{\lambda} \simeq \mathrm{k} \text { (cf. } \S \text { III.2) }
$$

The first term of (43) has the form of a derivative of $W:$ it is of the order of $\Delta v^{-2}$ and it can be neglected in comparison with the second term which is of the order of $\left(\Gamma_{a b}^{\prime} \Delta v\right)^{-1}$. In this second term $W$ varies only slowly over the range of values where the denominator is resonant. Finally one gets approximately :

$$
\begin{aligned}
(43)= & 2 \Delta v^{-1}\left[\Gamma_{b}^{\prime}(k)+i\left(\omega_{v}-\omega_{\mu}+Q \omega_{b}\right)\right]^{-1} \times \\
& \times\left[2 \Gamma_{a b}^{\prime}+i\left(\omega_{\kappa}-\omega_{\mu}+\left(q_{2}-q_{4}\right) \omega_{b}\right)\right]^{-1} \\
& \times X\left[\omega-\frac{1}{2}\left(\omega_{\mu}+\omega_{\kappa}-\left(q_{2}+q_{4}\right) \omega_{b}\right)\right] .
\end{aligned}
$$

With the approximation (42), $X(\Omega)$ which has been defined in (38), is simply the Gaussian Doppler line shape $\sqrt{\pi} \exp (\Omega / \Delta v)^{2}$.

The contribution of atoms which have experienced velocity changes at second order is obtained from the second term of $b_{Q}^{k}(v, v-\mu)$ and $a(v, v-\mu)((34)$ and (35) reported in (41)). As in our model there is no correlation between the velocity before and after a diffusion process, the denominators from first and from third orders are integrated separately over $v$. We get the product of two simple integrals of the form (38) :

$$
\begin{aligned}
\left\{\left[\Gamma_{b}(k)+i\left(\omega_{v}-\omega_{\mu}+Q \omega_{b}\right)\right]^{-1}-\left[\Gamma_{b}^{\prime}(k)\right.\right. & \left.\left.+i\left(\omega_{v}-\omega_{\mu}+Q \omega_{b}\right)\right]^{-1}\right\}\left[W\left(\omega-\omega_{v}-q_{1} \omega_{b}+i \Gamma_{a b}^{\prime}\right)\right. \\
& \left.+W^{*}\left(\omega-\omega_{\mu}+q_{2} \omega_{b}+i \Gamma_{a b}^{\prime}\right)\right] W\left(\omega-\omega_{k}+q_{4} \omega_{b}+i \Gamma_{a b}^{\prime}\right) .
\end{aligned}
$$

The first factor is resonant when $\omega_{v}+q_{1} \omega_{b} \simeq \omega_{\mu}-q_{2} \omega_{b}$, therefore the last factors can be written approximately

$$
\left(\frac{2}{\Delta v}\right) X\left(\omega-\omega_{v}+q_{1} \omega_{b}\right) \times W\left(\omega-\omega_{\kappa}+q_{4} \omega_{b}+i \Gamma_{a b}\right) .
$$

Furthermore it is possible to show that the imaginary part of the last $W$ cancels in the summation over $v, \mu, \kappa$ and $\lambda$. Thus (45) reduces to :

$$
\begin{aligned}
(45) \simeq\left\{\left[\Gamma_{b}(k)+i\left(\omega_{v}-\omega_{\mu}+Q \omega_{b}\right)\right]^{-1}-\left[\Gamma_{b}^{\prime}(k)+\right.\right. & \left.\left.i\left(\omega_{v}-\omega_{\mu}+Q \omega_{b}\right)\right]^{-1}\right\} \times \\
& \times 2 \Delta v^{-2} X\left(\omega-\omega_{v}+q_{1} \omega_{b}\right) X\left(\omega-\omega_{\kappa}+q_{4} \omega_{b}\right) .
\end{aligned}
$$

Each of the terms (46) is of the order of $\gamma_{b}^{\prime} /\left(\Gamma_{b} \Gamma_{b}^{\prime} \Delta v^{2}\right)$ and is small compared to (44) at resonance. However we must keep these terms because they are numerous and their sum may be important. Indeed for a given pair of modes $v, \mu$ we must sum over the $N-|v-\mu|$ possible values of $\kappa$ which provide terms of the same order of magnitude. On the other hand, the main term (44) is resonant only for one value of $\kappa$ if $\Delta \omega>\Gamma_{a b}^{\prime}$, or for $n \sim \Gamma_{a b}^{\prime} / \Delta \omega$ values if $\Gamma_{a b}^{\prime} \gtrsim \Delta \omega$ (far from resonance, the real part of (43), which will remain alone in the following, is as small as $\left.\Gamma_{a b} \Delta v^{-3}\right)$. Therefore, if the $N$ modes cover all the Doppler width $(N \Delta \omega \sim \Delta v)$ the ratio $\sum_{\kappa}(46) / \sum_{\kappa}(44)$ is of the

$$
\begin{aligned}
S=\frac{n\left|P_{a b}\right|^{4}}{9 \Delta v} & \sum_{v p s} \mathcal{R} e\left(\mathcal{E}^{v^{*}} \mathcal{E}^{v-p} \mathcal{E}^{v-p-s^{*}} \mathcal{E}^{v-s}\right) \times \\
& \times \operatorname{Re}\left\{2 \mathcal{J}^{\prime}(p)\left[C_{\mathrm{R}}(p-s) X\left(\delta_{v}-\frac{P+S}{2}\right)+\gamma_{b}^{\prime}(2) \Delta v^{-1} 3(p) X\left(\delta_{v}-\frac{P}{2}\right) X\left(\delta_{v}-S-\frac{P}{2}\right)\right]\right. \\
& +2 f_{1}^{\prime}(p) C_{\mathrm{R}}(p-s) X\left(\delta_{v}-\frac{P+S}{2}\right)+2 \Delta v^{-1}\left[f_{1}(p)-f_{1}^{\prime}(p)\right] X\left(\delta_{v}-\frac{P}{2}-\omega_{b}\right) X\left(\delta_{v}-s-\frac{P}{2}+\omega_{b}\right) \\
& +f_{2}^{\prime}(p) C(p-s)\left[X\left(\delta_{v}-P+\omega_{b}\right)+X\left(\delta_{v}-P-\omega_{b}\right)\right]+\Delta v^{-1}\left[f_{2}(p)-f_{2}^{\prime}(p)\right] \\
& \left.\times\left[X\left(\delta_{v}-\frac{P}{2}-\omega_{b}\right) X\left(\delta_{v}-\frac{P}{2}-S-\omega_{b}\right)+X\left(\delta_{v}-\frac{P}{2}+\omega_{b}\right) X\left(\delta_{v}-\frac{P}{2}-S+\omega_{b}\right)\right]\right\}
\end{aligned}
$$


with the " optical coincidence factors "

$$
C_{\mathrm{R}}(p-s)=\left[2 \Gamma_{a b}^{\prime}+i\left(P-S+2 \omega_{b}\right)\right]^{-1} ; \quad C(p-s)=\left[2 \Gamma_{a b}^{\prime}+i(P-S)\right]^{-1}
$$

and with the "Zeeman factors" :

$$
\begin{aligned}
\mathcal{J}^{\prime}(p)=\left[\Gamma_{b}^{\prime}(2)\right. & \left.+i\left(P+2 \omega_{b}\right)\right]^{-1} ; \quad 3(p)=\left[\Gamma_{b}(2)+i\left(P+2 \omega_{b}\right)\right]^{-1} \\
f_{\varepsilon}^{\prime}(p)=\left(\Gamma_{a}^{\prime}+i P\right)^{-1}\left[1-\gamma_{b a}\left(\Gamma_{b}^{\prime}(0)\right.\right. & \left.+i P)^{-1}\right] \\
& +\frac{1}{3}\left[\Gamma_{b}^{\prime}(0)+i P\right]^{-1}+(-)^{\varepsilon} \frac{1}{2}\left[\Gamma_{b}^{\prime}(1)+i P\right]^{-1}+\frac{1}{6}\left[\Gamma_{b}^{\prime}(2)+i P\right]^{-1}
\end{aligned}
$$

$\varepsilon=1$ or 2 refers to cases $a$ or $b$ of Table I. $f_{\varepsilon}(p)$ and $3(p)$ are obtained from $f_{\varepsilon}^{\prime}(p)$ and $\zeta^{\prime}(p)$ by using $\Gamma_{\alpha}(k)$ instead of $\Gamma_{\alpha}^{\prime}(k)$. Notice that

$$
3(p)-3^{\prime}(p)=\gamma_{b}^{\prime}(2) 3(p) 3^{\prime}(p) .
$$

In (48) the factorization of $\operatorname{Re}\left(\mathcal{E}^{*} \varepsilon^{\mu} \varepsilon^{\lambda^{*}} \varepsilon^{\kappa}\right)$ and the form used for $X$ functions have been obtained by applying some symmetry properties of the summation over $v, p, s$ and over $q_{i}$ 's.

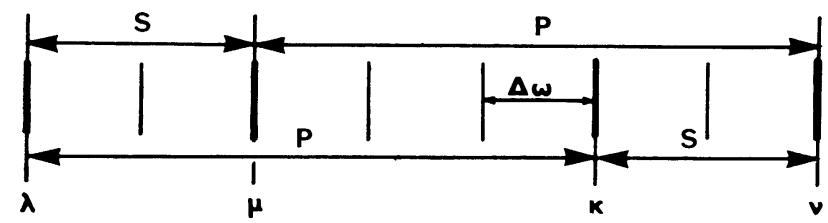

Fig. 3. - Modes in the frequency scale : definition of the notation. The condition $\omega_{\nu}-\omega_{\mu}+\omega_{\lambda}-\omega_{\kappa}=0$ insures the fourth order terms to be un-modulated. Modes are assumed bo be equidistant.

IV. Physical interpretation of the results. 1) THE CONTRIBUtion OF ATOMS WHOSE iVELOCITY HAS NOT CHANGED (TERMS LIKE (44)). - The first factor $\left[\Gamma_{b}^{\prime}(k)+i\left(\omega_{v}-\omega_{\mu}+Q \omega_{b}\right)\right]^{-1}$ expresses the ability of modes $v$ and $\mu$ to produce, at second order, the tensorial quantity ${ }_{b} T_{Q}^{k}$, modulated at frequency $\boldsymbol{P}=\omega_{v}-\omega_{\mu}$. Therefore this factor is resonant when the Zeeman splitting is equal to the beat frequency and we call it the "Zeeman factor » (cf. (50) and (51)). As we are concerned only with atoms whose velocity has not changed, the width of the resonance is $\Gamma_{b}^{\prime}(k)$.

The second factor in (44),

$$
\left\{2 \Gamma_{a b}^{\prime}+i\left[\omega_{\kappa}-\omega_{\mu}+\left(q_{2}-q_{4}\right) \omega_{b}\right]\right\}^{-1},
$$

is resonant when the $-q_{2}$ polarized component of mode $\mu$ and the $-q_{4}$ polarized component of mode $\kappa$ interact with atoms of the same velocity :

$$
\omega_{\mu}-q_{2} \omega_{b}=\omega_{\kappa}-q_{4} \omega_{b} .
$$

It expresses the need of an optical coincidence, for the same class of atomic velocity, between the first pair of modes $(\nu, \mu)$ and the second one $(\kappa, \lambda)$. The condition $\omega_{v}^{\prime}-\omega_{\mu}+\omega_{\lambda}-\omega_{\kappa}=0$ imposes the frequency of the fourth mode $\lambda$, and insures the second pair to have the exact beat frequency to demodulate ${ }^{(2)} \rho_{Q}^{k}\left(\omega_{v}-\omega_{\mu}\right)$. Finally, the third factor in (44), $X(\mathrm{)})$, is the Doppler distribution of atomic frequencies : it expresses the proportion of atoms which have the correct velocity to interact with the four modes.

It is now interesting to discuss separately the three cases of Table I, as they have been developed in (48).

Case $a$ : the Resonant Population Effect (PE). This case, and the symmetric on (all $q$ of opposite sign and the names of modes being exchanged: $v \leftrightarrow \lambda, \mu \leftrightarrow \kappa)$ represent the interaction of modes $\left(v, \sigma^{+}\right),\left(\mu, \sigma^{+}\right),\left(\lambda, \sigma^{-}\right)$and $\left(\kappa, \sigma^{-}\right)$. In (48) the corresponding term has the form

$$
\begin{aligned}
& \mathcal{R} e\left\{\mathcal{E}^{v *} \mathcal{E}^{\mu} \mathcal{E}^{\lambda^{*}} \mathcal{E}^{\kappa}\right\} \mathcal{R} e\{\left.f_{1}^{\prime}(p) C_{R}(p-s)\right\} \times \\
& \times X\left(\delta_{v}-\frac{1}{2}(P+S)\right) .
\end{aligned}
$$

Included in $f_{1}^{\prime}(p)$, the «Zeeman factors » $\left[\Gamma_{\alpha}^{\prime}(k)+i P\right]^{-1}$ do not depend on the magnetic field : the second order is composed of longitudinal quantities $(Q=0)$ or, in other words, of populations of the Zeeman sublevels. It is produced by beating of the two $\sigma^{+}$ modes $(v$ and $\mu) . f_{1}^{\prime}(p)$ is resonant for $p=0$ $(v=\mu ; \kappa=\lambda)$ : most often $\Gamma_{b}^{\prime}(k)$ is smaller than the mode spacing $\Delta \omega$ and one can neglect $f_{1}^{\prime}(p)$ for $p \neq 0$. Therefore (52) becomes

$$
\begin{aligned}
& \left|\mathcal{E}^{\mu}\right|^{2}\left|\mathcal{E}^{\kappa}\right|^{2} X\left(\frac{1}{2}\left(\delta_{\mu}+\delta_{\kappa}\right)\right) \times \\
& \quad \times f_{1}^{\prime}(0) 2 \Gamma_{a b}^{\prime}\left[4 \Gamma_{a b}^{\prime 2}+\left(\omega_{\kappa}-\omega_{\mu}+2 \omega_{b}\right)^{2}\right]^{-1}
\end{aligned}
$$

The Lorentzian resonance arising from the " optical coincidence factor $», C_{R}(p-s)$, expresses the fact that there is a maximum of saturation when the $\left(\mu, \sigma^{+}\right)$and the $\left(\kappa, \sigma^{-}\right)$modes interact with atoms of the same velocity. The scanning of the resonance corresponds to the crossing of holes burned [5] by these modes in the Doppler distribution (Fig. 4a). The width of the resonance is two times the width $\Gamma_{a b}^{\prime}$ of holes. This phenomenon is the intuitive magnetic mode crossing effect. 

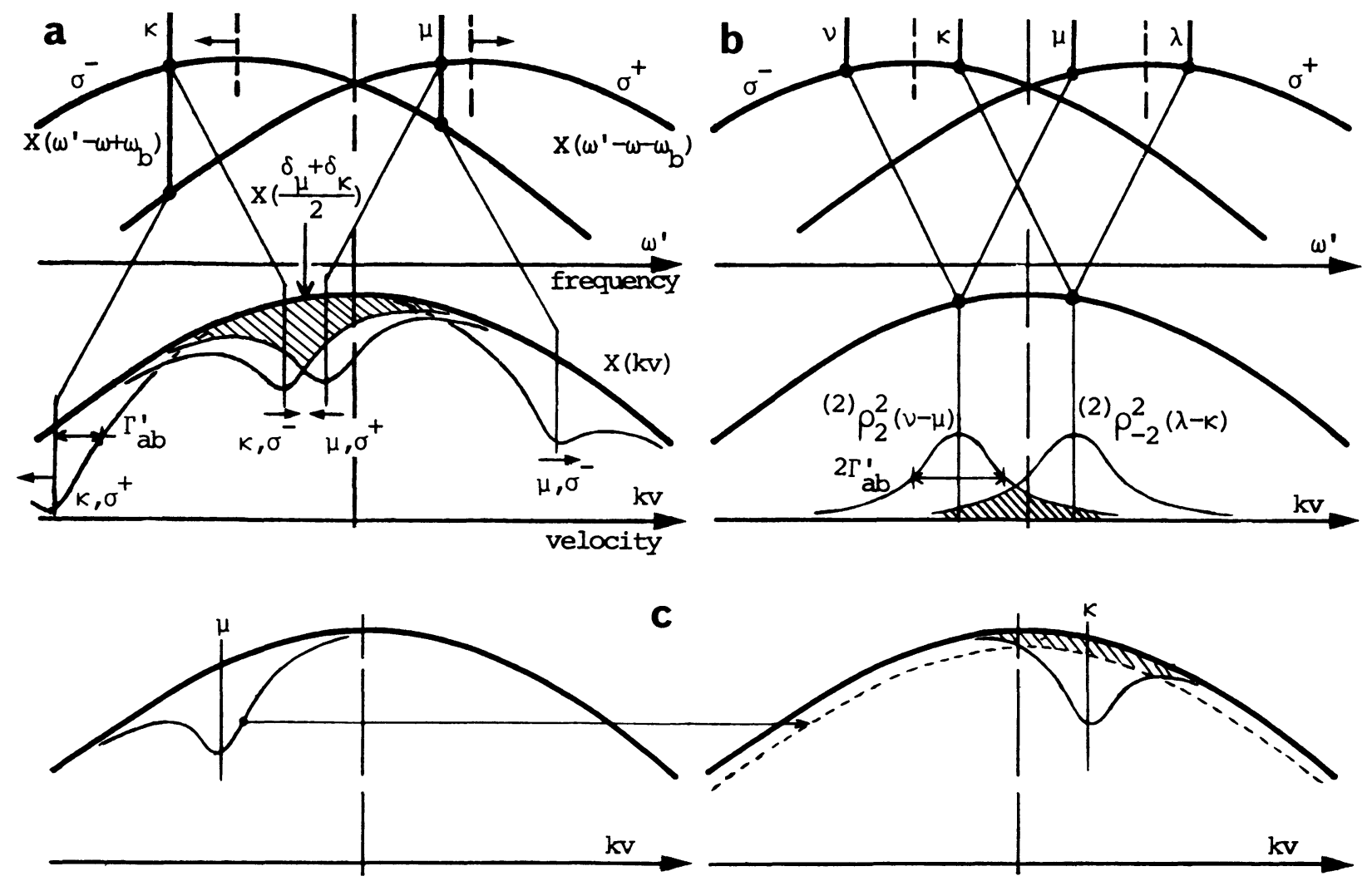

Fig. 4. - Interpretation of the different kind of saturation terms :

4a. - The saturation by "Population Effect». Holes represent the effect of each mode if it were alone (effect at second order of perturbation). The overlapping of two holes gives an idea of the magnitude of the non linear interaction. The arrows indicate the sense of displacement of holes when the magnetic field increases. The PE resonance is due to the crossing of $\left(\kappa, \sigma^{-}\right)$and $\left(\mu, \sigma^{+}\right)$holes.

4b. - Velocity distribution, at second order, of the transverse alignment modulated at frequencies $\left(\omega_{\nu}-\omega_{\mu}\right)$ and $\left(\omega_{\lambda}-\omega_{\kappa}\right)=\omega_{\mu}-\omega_{\nu}$. This drawing corresponds to exact resonance of the Zeeman factor. The overlapping of the two distributions is related to the magnitude of the fourth order term which contributes to the "Zeeman Coherence Effect ».

4c. - Saturation arising from atoms whose velocity has changed at second order: this drawing is concerned with the special case of population at second order $\left(v=\mu ; q_{1}=-q_{2} ; \kappa=\lambda ; q_{3}=-q_{4}\right)$. The diminution of population, primitively produced by modes $\mu$ and $v$ as a narrow dip, is then distributed all over the Doppler profile. The dashed part shows the proportion of atoms (among those which have first interact with $\mu$ and $v$ ) which can interact with a given second pair of modes $(\kappa, \lambda)$ at third and fourth order.

Case $b$ : the Non Resonant Population Effect. This case produces terms of the form

$$
\begin{aligned}
& X\left(\delta_{v}-P \pm \omega_{b}\right)\left[\Gamma_{\alpha}^{\prime}(k)+i P\right]^{-1} \times \\
& \times\left[2 \Gamma_{a b}^{\prime}+i(P-S)\right]^{-1} .
\end{aligned}
$$

It depends on the magnetic field only by the slowly varying Doppler function $X(\quad)$. It corresponds to the interaction of four $\sigma^{-}$(or four $\sigma^{+}$) components. For $P=0$ (the most important terms) it can be interpreted by the overlapping of holes $\left(\mu, \sigma^{+}\right)$and $\left(\kappa, \sigma^{+}\right)$or of $\left(\mu, \sigma^{-}\right)$and $\left(\kappa, \sigma^{-}\right)$on figure $4 a$.

Case $c$ : the "Zeeman Coherence Effect» $(Z C E)$. This case and the symmetric one (Table I) produce terms of the form :

$$
\begin{array}{r}
\operatorname{Re}\left\{\mathcal{E}^{v^{*}} \mathcal{E}^{\mu} \mathcal{E}^{\lambda^{*}} \mathcal{E}^{\kappa}\right\} X\left(\frac{1}{2}\left(\delta_{\mu}+\delta_{\kappa}\right)\right)\left\{\left[\Gamma_{b}^{\prime}(2)+i\left(\omega_{v}-\omega_{\mu}+2 \omega_{b}\right)\right]^{-1}\left[2 \Gamma_{a b}^{\prime}+i\left(\omega_{\kappa}-\omega_{\mu}+2 \omega_{b}\right)\right]^{-1}+\right. \\
\left.+\left[\Gamma_{b}^{\prime}(2)+i\left(\omega_{\lambda}-\omega_{\kappa}-2 \omega_{b}\right)\right]^{-1}\left[2 \Gamma_{a b}^{\prime}+i\left(\omega_{\mu}-\omega_{\kappa}-2 \omega_{b}\right)\right]^{-1}\right\}= \\
=\operatorname{Re}\left\{\mathcal{E}^{v^{*}} \mathcal{E}^{\mu} \mathcal{E}^{\lambda^{*}} \mathcal{E}^{\kappa}\right\} X\left(\delta_{v}-\frac{1}{2}(P+S)\right)\left\{\zeta^{\prime}(p) C_{p}(p-s)+c c\right\}
\end{array}
$$


As appears from the denominator

$$
\Gamma_{b}^{\prime}(2)+i\left(\omega_{v}-\omega_{\mu}+2 \omega_{b}\right)
$$

the first term of (55) arises from the transverse alignment ${ }_{b}^{(2} \rho_{2}^{2}$ modulated at frequency $\omega_{v}-\omega_{\mu}$ by the action at the two first orders, of

$$
\varepsilon_{\sigma^{-}}^{v^{*}} \exp \left(i \omega_{\nu} t\right) \text { and } \varepsilon_{\sigma^{+}}^{\mu} \exp \left(-i \omega_{\mu} t\right) .
$$

Thereafter the fourth order is obtained by applying

$$
\varepsilon_{\sigma^{+}}^{\lambda^{*}} \exp \left(i \omega_{\lambda} t\right) \text { and } \varepsilon_{\sigma^{-}}^{\kappa} \exp \left(-i \omega_{\kappa} t\right)
$$

which remove the modulation. In the second terms of (55), the second order quantity ${ }_{h}^{(2)} \rho_{-2}^{2}$, modulated at $\omega_{\lambda}-\omega_{\kappa}=-\left(\omega_{v}-\omega_{\mu}\right)$ by prior action of $\left(\lambda, \sigma^{+}\right)$ and $\left(\kappa, \sigma^{-}\right)$, is demodulated by $\left(\nu, \sigma^{-}\right)$and $\left(\kappa, \sigma^{+}\right)$. When $v=\mu$ and $\kappa=\lambda$ the resonance in zero magnetic field of the Zeeman factor is simply the Hanle effect due to unmodulated transverse alignment at second order. The optical coincidence factor, $C_{R}(p-s)$, which expresses the possibility for the two pairs to interact with the same atoms, is difficult to interpret graphically. It is necessary to replace the idea of holes burned in the velocity distribution of populations, by the idea of velocity distribution of atoms which carry transverse alignment modulated at $\omega_{v}-\omega_{\mu}$. When the Zeeman factor is exactly resonant $\left(\omega_{v}-\omega_{\mu}=-2 \omega_{b}\right)$ this velocity distribution is Lorentzian with a width $\Gamma_{a b}^{\prime}$ exactly like holes in the previous case. Then the optical coincidence factor expresses the overlapping of the velocity distributions of

$$
\left.{ }_{b}^{(2)} \rho_{2}^{2}\left(\omega_{v}-\omega_{\mu}\right) \text { and of }{ }_{b}^{(2)} \rho_{-2}^{2}\left(\omega_{\lambda}-\omega_{\kappa}\right) \text { (Fig. } 4 b\right) \text {. }
$$

When the magnetic field is scanned it is not easy to follow graphically the evolution of the velocity distribution which are distorted and which acquire an imaginary part.

2) THE CONTRIBUtion OF ATOMS WHOSE VELOCITY HAS CHANGED AT SECOND ORDER. - Like (44), (46) contains a first factor which expresses the resonances of atomic quantities at second order (Zeeman factor), but this factor is more complicated ; it can be written (for $b$ level) :

$$
\begin{aligned}
\gamma_{b}^{\prime}(k)\left[\Gamma_{b}^{\prime}(k)\right. & \left.+i\left(\omega_{v}-\omega_{\mu}+Q \omega_{b}\right)\right]^{-1} \times \\
& \times\left[\Gamma_{b}(k)+i\left(\omega_{v}-\omega_{\mu}+Q \omega_{b}\right)\right]^{-1} .
\end{aligned}
$$

The last factor expresses the evolution of ${ }_{b}^{(2)} \bar{\rho}_{Q}^{k}(v-\mu)$ before the last velocity diffusion process : as the initial velocity does not matter, the width is $\Gamma_{b}(k)$ (insensitive to velocity changes). $\gamma_{b}^{\prime}(k)$ is the probability that this last process leaves the atom in the considered velocity class. Finally, the factor with $\Gamma_{b}^{\prime}(k)$ (relaxation rate including velocity changes) expresses the evolution after the last diffusion process up to a new interaction with the laser beam. In the $a$ level the corresponding expression is complicated by spontaneous emission from $b$ level.

On the contrary to (44), (46) contains no resonant optical factor of width $2 \Gamma_{a b}^{\prime}$ : it is replaced by a second $X$ factor. This is easy to understand with the model used for velocity diffusion processes : atoms lose all memory of their initial velocity, therefore the first pair of modes $(\nu, \mu)$ and the second one $(\kappa, \lambda)$ have no need to interact with the same class of velocities. When the Zeeman factor is resonant, modes $\left(v, q_{1}\right)$ and $\left(\mu,-q_{2}\right)$ interact with atoms, located in a $2 \Gamma_{a b}^{\prime}$ frequency width band, whose number is $n\left(\Gamma_{a b}^{\prime} / \Delta v\right) X\left(\delta_{v}+q_{1} \omega_{b}\right)$. Due to collisions or trapping of lines, some of these atoms (probability $\gamma_{b}^{\prime}(k)$ ) are randomly distributed according to Maxwellian distribution (Fig. 4c). Among them the proportion $\left(\Gamma_{a b}^{\prime} / \Delta v\right) X\left(\delta_{\kappa}-q_{4} \omega_{b}\right)$ gets a final velocity which allows them to interact with modes $\left(\lambda, q_{3}\right)$ and $\left(\kappa,-q_{4}\right)$. It is easy to understand why the contribution of atoms whose speed has changed becomes important when the modes are numerous enough $(N \Delta \omega \gtrsim \Delta v)$ and close enough $\left(\Gamma_{a b}^{\prime} \gtrsim \Delta \omega\right)$ to cover all the Doppler line shape : every atom which has interacted with modes $v$ and $\mu$ is certain, whatever its final velocity is, to find a second pair $(\kappa, \lambda)$ to interact again.

V. Relative amplitude of saturation resonances. The eq. (48) shows that the saturation signal, observable on the fluorescent light detected with a $\pi$ analyser, exhibits resonances when the magnetic field is scanned $\left(\omega_{b}=\beta g_{b} H\right)$. We shall study the behaviour of these resonances for different structures of modes.

1) LARGE MODE SPACING. - Many simplifications occur when the mode spacing is much larger than the hole width, i. e. when

$$
\Gamma_{\alpha}^{\prime}, \Gamma_{a b}^{\prime} \ll \Delta \omega \text {. }
$$

First we can neglect the effect of velocity diffusion which is of the order of $\Gamma_{a b}^{\prime} / \Delta \omega$ as explained in paragraph III.4. Secondly, in the first term of (48) (ZCE, cf. eq. (55)) we keep only the value $s=0(v=\kappa$, $\mu=\lambda$ ) for which the two denominators are simultaneously resonant. This means, on figure $4 b$, that the two distributions do not overlap, except if they exactly coincide. Third, for the resonant population effect (terms like (52)), we must choose $p=0$ and keep expressions of the form (53). Finally, omitting non resonant terms (54), we get

$$
\begin{aligned}
& S_{\mathrm{res}} \propto \sum_{v r}\left|\varepsilon^{v}\right|^{2}\left|\mathcal{E}^{v-r}\right|^{2} X\left(\delta_{v}-\frac{r \Delta \omega}{2}\right) \times \\
& \times {\left[\operatorname{Re}\left(\frac{1}{\Gamma_{b}^{\prime}(2)+i\left(r \Delta \omega+2 \omega_{b}\right)} \frac{1}{2 \Gamma_{a b}^{\prime}+i\left(r \Delta \omega+2 \omega_{b}\right)}\right)+f_{1}^{\prime}(0) \frac{2 \Gamma_{a b}^{\prime}}{4 \Gamma_{a b}^{\prime 2}+\left(r \Delta \omega+2 \omega_{b}\right)^{2}}\right] }
\end{aligned}
$$


where $r$ is put or $p$ in the first term and for $-s$ in the second. It is interesting to notice that :

Each resonance (arising for $r \Delta \omega=-2 \omega_{b}$ ) is composed of two terms : one is Lorentzian of $2 \Gamma_{a b}^{\prime}$ width and comes from the PE. The other which has a more complicated shape, is due to the ZCE; when $\Gamma_{b}^{\prime}(2) \ll \Gamma_{a b}^{\prime}$, as it is usually, its width is almost the relaxation rate of alignment $\Gamma_{b}^{\prime}(2)$ (including velocity changes).

- (58) depends only on the intensity of modes $\left(I_{v}=\left|\mathcal{E}_{v}\right|^{2}\right)$ : as the only important terms involve no more than two different modes, the phases are cancelled out. If modes frequencies are not equidistant each resonance is split, but in general this splitting (few $\mathrm{kHz}$ ) is negligible in comparison with the width (few $\mathrm{MHz}$ ).

- The amplitude of the $r$ th resonance is proportional to $\sum_{v} I_{v} I_{v-r}$ : it decreases regularly from the zero field resonance $(r=0)$ to the high field resonances.

- (58) is valid for a monomode laser (only one resonance for $\omega_{b}=0$ ) provided that the model used for relaxation is still valid.

It appears that (58) cannot explain the experimental behaviour of resonances when the modes are not phase locked.

2) MOde SPACING OF THE ORDER OF HOLES WIDTH. - We consider now the case

$$
\Gamma_{\alpha}^{\prime}(k)<\Gamma_{a b}^{\prime} \sim \Delta \omega .
$$

This is the usual conditions in our experiments ( $\Delta \omega \simeq 80 \mathrm{MHz}$, pressure of the order of a few torr). It is no longer possible to neglect terms arising from velocity diffusion nor those which contain two denominators which are resonant in different magnetic fields.

Let us first study terms arising from $\mathrm{ZCE}$, without velocity change (eq. (55)). For each value of $p$ it is now necessary to sum over all possible values of $s$. As the Zeeman factor (width $\Gamma_{b}^{\prime}(2)$ ) is narrower than the optical one (width $2 \Gamma_{a b}$ ) it imposes the position of resonances. Therefore the resonance near $p \Delta \omega=2 \omega_{b}$ can be written in the symmetrical form (by permutation of modes indexes) :

$$
\begin{aligned}
\operatorname{Res}(p)=\sum_{v s} \frac{1}{2} X\left(\delta_{v}-\frac{P+S}{2}\right) & \frac{\operatorname{Re}\left(\mathcal{E}^{v^{*}} \mathcal{E}^{v-p} \mathcal{E}^{v-p-s^{*}} \mathcal{E}^{v-s}\right)}{\Gamma_{b}^{\prime}(2)+i\left(P+2 \omega_{b}\right)} \times \\
\times & {\left[\frac{1}{2 \Gamma_{a b}^{\prime}+i\left(P-S+2 \omega_{b}\right)}+\frac{1}{2 \Gamma_{a b}^{\prime}+i\left(P+S+2 \omega_{b}\right)}\right]+c c . }
\end{aligned}
$$

In this form it is easy to see that the resonances remain symmetric with respect to the center $p \Delta \omega=-2 \omega_{b}$ and that terms with $s \neq 0$ decrease only slowly when $s$ increases :

$$
\text { amplitude at resonance } \propto\left[1+\left(s \Delta \omega / 2 \Gamma_{a b}^{\prime}\right)^{2}\right]^{-1} \text {. }
$$

The resonance in zero magnetic field is obtained for $p=0$, i. e. for $v=\mu$ and $\kappa=\lambda$. As each term involves only two modes, it depends only upon the intensity of modes and the amplitude of the resonance is of the order of (if we assume $N$ modes of approximately equal intensity) :

$$
\begin{aligned}
& \operatorname{Res}(p=0) \simeq I_{v}^{2}\left(\Gamma_{b}^{\prime}(2) \Gamma_{a b}^{\prime}\right)^{-1} \times \\
& \times\left\{N+\sum_{s=1}^{N} 2(N-s)\left[1+\left(\frac{s \Delta \omega}{2 \Gamma_{a b}^{\prime}}\right)^{2}\right]^{-1}\right\} .
\end{aligned}
$$

On the contrary, for resonances in non zero magnetic field $(p \neq 0)$, all terms with $s \neq 0$ involve more than two modes and they are sensitive to the relative phases of modes :

$$
\begin{gathered}
\operatorname{Re}\left(\mathcal{E}^{v^{*}} \mathcal{E}^{v-p} \mathcal{E}^{v-p-s^{*}} \mathcal{E}^{v-s}\right)=\sqrt{I_{v} I_{v-p} I_{v-p-s} I_{v-s}} \times \\
\times \cos \left(\varphi_{v}-\varphi_{v-p}+\varphi_{v-p-s}-\varphi_{v-s}\right)
\end{gathered}
$$

When modes are free running, their phases are randomly distributed. Moreover, modes are in general not exactly equidistant and the relative phases can be regarded as slowly varying (we can write

$$
\left.\omega_{v} t+\phi_{v}=\left(\omega_{0}+v \Delta \omega\right) t+\phi_{v}(t)\right) .
$$

Therefore, except for $s=0$, (62) cancels when averaged (sum over $v$ and average over time). Therefore, the amplitude of the $p$ th resonance is of the order of

$\operatorname{Res}(p \neq 0)($ free running $) \simeq$

$$
\simeq I_{v}^{2}\left(\Gamma_{b}^{\prime}(2) \Gamma_{a b}^{\prime}\right)^{-1}(N-p) \text {. }
$$

When modes are phase locked in such a manner that they are all in phase at regular times intervals (the laser is then modulated in short repetitive pulses), it is easy to show that all relative phases are equal for a correct choice of the origin of time. Therefore the cosine of (62) is equal to one in every case, and the amplitude of the resonance is of the order of :

$\operatorname{Res}(p \neq 0)($ phase locked $) \simeq I_{v}^{2}\left(\Gamma_{b}^{\prime}(2) \Gamma_{a b}^{\prime}\right)^{-1} \times$

$$
\times\left\{(N-p)+\sum_{s=1}^{N-p} 2(N-p-s)\left[1+\left(\frac{s \Delta \omega}{2 \Gamma_{a b}^{\prime}}\right)^{2}\right]^{-1}\right\}
$$


It appears that, when the mode spacing $\Delta \omega$ is of the order of the optical line width $\Gamma_{a b}^{\prime}$, the resonances due to the ZCE are much more important when the modes are phase locked than when they are free running. Only the resonance in zero magnetic field is unaffected and remains always important. The conclusion is the same for the terms arising from ZCE with velocity changes at second order.

Finally the resonances of the form (52), arising from population effects, are not observable since they all overlap, due to their broad width $2 \Gamma_{a b}^{\prime}$. Further- more the terms sensitive to the phase of modes (terms $p \neq 0)$ remain small as long as $\Gamma_{\alpha}(k)$ is smaller than $\Delta \omega$.

It is interesting to notice that, when $\Gamma_{a b}^{\prime} \sim \Delta \omega$, the saturation resonances observed are due only to the Zeeman coherence effect and no longer to the intuitive population effect.

Numerical calculation. - To illustrate these results, we have performed a computer calculation of the ZCE resonances (including velocity changes) :

$$
\begin{aligned}
& A(p, s)=\sum_{v} \frac{\sqrt{I_{v} I_{v-p} I_{v-s-p} I_{v-s}}}{\Gamma_{b}^{\prime}(2)+i\left(p \Delta \omega+2 \omega_{b}\right)}\left\{\frac{X\left(\delta_{v}-\frac{p+s}{2} \Delta \omega\right)}{2 \Gamma_{a b}^{\prime}+i\left((p-s) \Delta \omega+2 \omega_{b}\right)}+\right. \\
& \left.+\frac{\gamma_{b}^{\prime}(2) X\left(\delta_{v}-\frac{p \Delta \omega}{2}\right) X\left(\delta_{v}-\left(s+\frac{p}{2}\right) \Delta \omega\right)}{\Gamma_{b}(2)+i\left(p \Delta \omega+2 \omega_{b}\right)}\right\}+c c
\end{aligned}
$$

and of the resonant $\mathrm{PE}$

$$
B(p, s)=\sum_{v} \sqrt{I_{v} I_{v-p} I_{v-p-s} I_{v-s}} f_{1}^{\prime}(p) X\left(\delta_{v}-\frac{p+s}{2} \Delta \omega\right)\left[2 \Gamma_{a b}^{\prime}+i\left((p-s) \Delta \omega+2 \omega_{b}\right)\right]^{-1}+c c .
$$

The used numerical values correspond approximately to the $7305 \AA$ laser line $\left(3 s_{2} \rightarrow 2 p_{1}\right)$ with a neon pressure of 1.5 torr (cf. Fig. 5 caption). The $3 s_{2}$ relaxation rates are deduced from Hanle effect experiments [1], assuming that velocity diffusion is due only to trapping of the fluorescence line connecting the $3 s_{2}$ level to the ground state. The estimated relaxation rate $\left(\Gamma_{a}=15 \mathrm{MHz}\right)$ of the $2 p_{1}$ level appears overestimated when compared with very new measurements [28] but this is of little importance for the final result. The order of magnitude of the optical

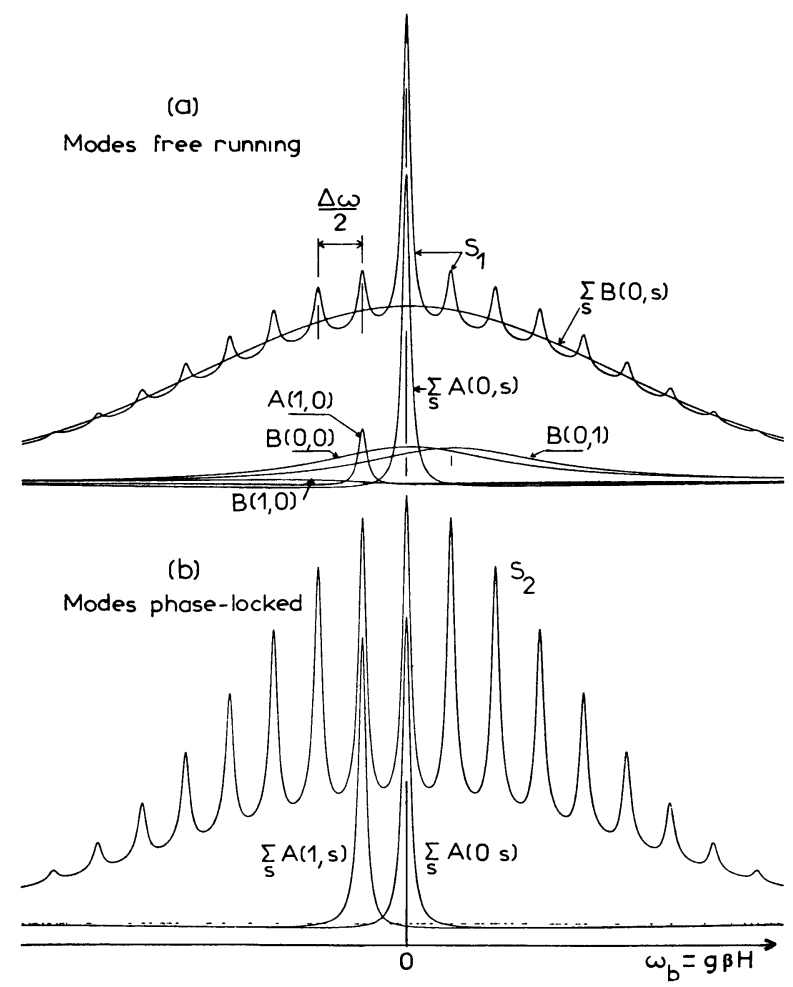

width $\left(\Gamma_{a b}^{\prime}=100 \mathrm{MHz}\right)$ has been estimated by comparison with Cordover's experiments [29] on the $6328 \AA$ laser line.

When modes are free running only terms with $p$ or $s=0$ are to be kept, as they involve only two modes. Figure $5 a$ shows a computer drawing of the different resonant terms and of the resulting total signal

$$
\begin{aligned}
S_{1}=\sum_{s}(A(0, s)+ & B(0, s))+ \\
& +\sum_{p \neq 0}(A(p, 0)+B(p, 0)) .
\end{aligned}
$$

FIG. 5. - Computer calculation of saturation resonances produced by 11 modes $\left(I_{v}=\varepsilon_{v}^{2}=3\right.$ for $-3 \leqslant v \leqslant 3, \varepsilon_{v}^{2}=2$ for $v= \pm 4, \varepsilon_{v}^{2}=1$ for $v= \pm 5$ ). The Doppler width is $\Delta \omega=800 \mathrm{MHz}$. The mode spacing $\Delta \omega=80 \mathrm{MHz}$ and the relaxation rates are (in $\mathrm{MHz}): \Gamma_{a b}^{\prime}=100 ; \Gamma_{b}^{\prime}(0)=9.6$; $\Gamma_{b}^{\prime}(1)=11 ; \Gamma_{b}^{\prime}(2)=11.5 ; \Gamma_{b}(2)=7,5 ; \Gamma_{a}^{\prime}=15$. These conditions correspond approximately to the laser line $7305 \AA$ with 1.5 torr of Neon (see Ref. [1]).

$5 a$. - Modes free running : resonances from Zeeman Coherence Effects and from Population Effects are compared. ZCE resonances are narrow and resolved; the resonance in zero magnetic field $\left(\sum_{s} A(0, s)\right)$ is mush higher than the others $(A(p, 0))$. PE resonances $(B(0,0)$ and $B(0,1))$ are not resolved as it is shown by the curve $\sum_{s} B(0, s)$. Off resonances terms $B(p, 0)$ are negligible. $S_{1}=\sum_{s \text { or } p=0}(A(p, s)+B(p, s))$ is the resulting signal.

5b. - Modes phase-locked : ZCE resonance for $p=1$ has the same order of magnitude than that in zero magnetic field. $S_{2}=\sum_{s p}(A(p, s)+B(p, s))$ is the total signal. 
On the other hand, when modes are phase locked all values of $s$ and $p$ are possible. As shown on figure $5 b$ the terms $A(p, s)$ produce an increase of the non zero magnetic field resonances. The total signal is then

$$
S_{2}=\sum_{s p}(A(p, s)+B(p, s)) \text {. }
$$

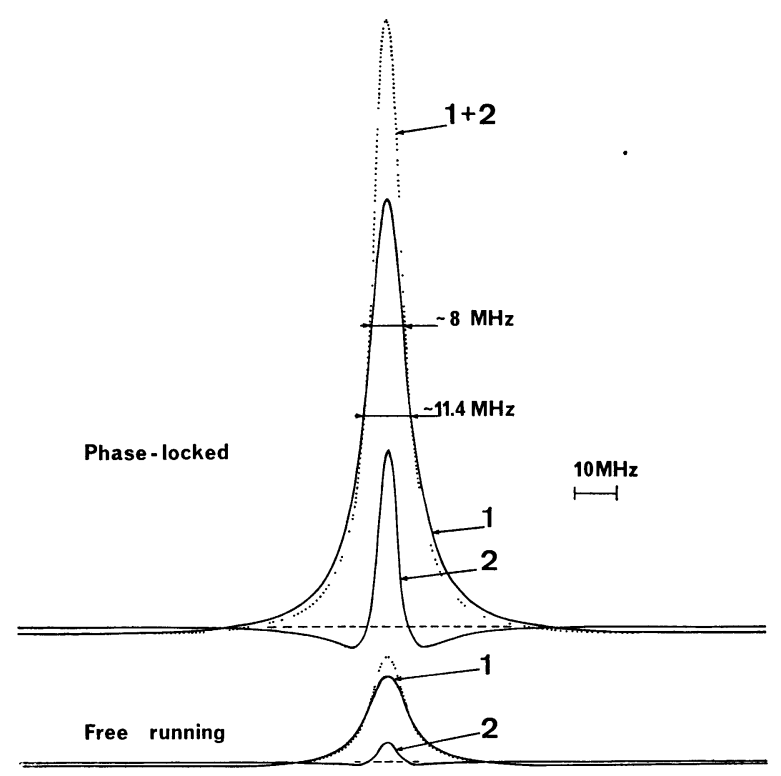

FIG. 6. - Computation of the ZCE resonance for $p=1$ : (1) contribution of atoms whose velocity has not changed; 2) contribution of atoms whose velocity has changed. Numerical values are the same as those used on figure 5. In the phase-locked case the width of (1) appears to be very close to $\Gamma_{b}^{\prime}(2)=11.5 \mathrm{MHz}$ and the width of the total resonance $(1)+(2)$ is close to $\Gamma_{b}(2)=7.5 \mathrm{MHz}$ (cf. eq. (71) and (74)).
Figure 6 shows separately the contribution to ZCE of atoms whose speed has changed (more precisely the effect of restitution of alignment by trapping of the fluorescence light).

Conclusion. - The comparison between figures $5 a$ and $5 b$ illustrates clearly the conclusions of the previous calculation on the effect of the phase of modes. These conclusions appear to be in good agreement with the experimental behaviour of resonances as they have been described in the introduction.

3) ANALYTICAL APPROXIMATION FOR VERY CLOSE MODES. - Let us consider the limiting case where modes are very close (many modes in one optical width) and very numerous (mode distribution width of the order of Doppler width), namely :

$$
\Gamma_{\alpha}(k)<\Delta \omega \ll \Gamma_{a b}^{\prime} \ll \Delta v \sim N \Delta \omega .
$$

In this case it is possible to use integrals instead of some of the summations, $I_{v}$ being considered as a continuous function of $\delta_{v}=\omega_{v}-\omega$, such as $I\left(\delta_{v}\right)=I_{v}$. Replacing

$$
\sum_{v} \text { by } \int \frac{\mathrm{d} \delta}{\Delta \omega} \text { and } \sum_{s} \text { by } \int \frac{\mathrm{d} S}{\Delta \omega},
$$

the resonance at $p \Delta \omega=2 \omega_{b}$ becomes (phase locked modes) :

$S_{p}$ (locked) $\propto A(p, s)$

$$
\begin{aligned}
& =\frac{\zeta^{\prime}(p)}{\Delta \omega^{2}} \int \sqrt{I(\delta) I(\delta-P)} \mathrm{d} \delta \int X\left(\delta-\frac{P+S}{2}\right) \sqrt{I(\delta-S) I(\delta-P-S)} C_{p}(p-s) \mathrm{d} S+ \\
& +\frac{3(p)-\zeta^{\prime}(p)}{\Delta v \Delta \omega^{2}} \int \sqrt{I(\delta) I(\delta-P)} X\left(\delta-\frac{P}{2}\right) \mathrm{d} \delta \int \sqrt{I(\delta-S) I(\delta-P-S)} X\left(\delta-S-\frac{P}{2}\right) \mathrm{d} S+c c .
\end{aligned}
$$

If $I(\delta)$ is a slowly varying function, like $X(\delta)$, they both may be taken out of the integral over $S$ in the first term, with the values they take for the peak of
$C_{\mathrm{R}}(p-s)$, i. e. for $S=P+2 \omega_{b} \simeq 0$ (as the $3^{\prime}(p)$ factor is resonant for $\left.p+2 \omega_{b}=0\right)$. Then we obtain the $p$ resonance shape (reintroducing all factors)

$$
\begin{aligned}
S_{p} \text { (locked) }=\frac{2}{9} n\left|P_{a b}\right|^{4} \Delta \omega^{-2}\left\{\pi \mathfrak{L}_{b}^{\prime}(p) \int I(\delta)\right. & I(\delta-P) X\left(\delta-\frac{P}{2}\right) \Delta v^{-1} \mathrm{~d} \delta+ \\
& \left.+\left(\mathfrak{L}_{b}(p)-\mathfrak{L}_{b}^{\prime}(p)\right)\left(\int \sqrt{I(\delta) I(\delta-P)} X\left(\delta-\frac{P}{2}\right) \Delta v^{-1} \mathrm{~d} \delta\right)^{2}\right\}
\end{aligned}
$$

with the Lorentzian curves :

$$
\mathfrak{L}_{b}^{\prime}(p)=\Gamma_{b}^{\prime}(2)\left(\Gamma_{b}^{\prime}(2)^{2}+\left(P+2 \omega_{b}\right)^{2}\right)^{-1} ; \quad \mathcal{L}_{b}(p)=\Gamma_{b}(2)\left(\Gamma_{b}(2)^{2}+\left(P+2 \omega_{b}\right)^{2}\right)^{-1} .
$$

When the modes are free running (71) is valid only for the central resonance $p=0$. For other resonances there is no integration over $s$; we obtain :

$$
\begin{aligned}
S_{p \neq 0} \text { (unlocked) } & =\frac{2}{9} n\left|P_{a b}\right|^{4} \Delta \omega^{-1}\left\{\operatorname{Re}\left\{\left(\Gamma_{b}^{\prime}(2)+i\left(P+2 \omega_{b}\right)\right)^{-1}\left(2 \Gamma_{a b}^{\prime}+i\left(P+2 \omega_{b}\right)\right)^{-1}\right\} \times\right. \\
\times & \left.\int I(\delta) I(\delta-P) X\left(\delta-\frac{P}{2}\right) \frac{\mathrm{d} \delta}{\Delta v}+\Delta v^{-1}\left[\mathcal{L}_{b}^{\prime}(p)-\mathcal{L}_{b}(p)\right] \int I(\delta) I(\delta-P) X^{2}\left(\delta-\frac{P}{2}\right) \frac{\mathrm{d} \delta}{\Delta v}\right\} .
\end{aligned}
$$


We find again the conclusion of the previous paragraph with some new results :

- When modes are free running the contribution of atoms whose velocity has changed appears clearly negligible (by a factor $2 \Gamma_{a b}^{\prime} / \Delta v$ ).

- For the main term, the $p$ resonance amplitude is higher in (71) than (73) by a factor $2 \pi \Gamma_{a b}^{\prime} / \Delta \omega$. This factor can be understood as the number of neighbour modes interacting with a given one, or in other words, the number of modes in the same «hole». For $\Delta \omega=80 \mathrm{MHz}$ and $\Gamma_{a b}=100 \mathrm{MHz}$, one finds $2 \pi \Gamma_{a b}^{\prime} \Delta \omega^{-1} \simeq 8$. With the same values, figure 6 gives only 5 as the conditions (69) are not perfectly satisfied in this realistic case.

- The resonances (71) (mode locked case) no longer depend on the optical width $\Gamma_{a b}^{\prime}$. They involve only Lorentzian curves of width $\Gamma_{b}^{\prime}(2)$ and $\Gamma_{b}(2)$. Furthermore if we assume $I(\delta)=I_{v}$ to be a constant over the wole Doppler line shape the integrals are easily calculated $\left(\Delta v^{-1} \int X(\delta) \mathrm{d} \delta=\pi\right)$ and (71) becomes :

$$
S_{p}(\text { locked })=\frac{2}{9} n \pi^{2} \Delta \omega^{-2}\left|P_{a b}\right|^{4} I_{v}^{2} \mathcal{L}_{b}(p) .
$$

The conditions needed for the validity of (74) are somewhat extreme but it is interesting to see that, at the limit of a great number of phase-locked modes, the resonances tend to be Lorentzian curves of width $\Gamma_{b}(2)$. That is to say that they tend to have exactly the shape of the Hanle effect [30] and to be insensitive to velocity changes. On figure 6 it is easy to see that these conclusions are verified within a few percent even with realistic numerical values.

On the other hand, for free running modes, resonances $p \neq 0$ are not exactly Lorentzian (73) and their width, which is near $\Gamma_{b}^{\prime}(2)$ (if $\Gamma_{a b}^{\prime} \gg \Gamma_{b}^{\prime}(2)$ ), is sensitive to velocity changes. Notice that (73) has exactly the shape of resonances for large mode spacing if we assume the second term of (54) to be flat enough.

The analytical method applied to $B(p, s)$ shows that the population effect produces no resonances as explained intuitively in the previous paragraph.

Remark. - It is interesting to notice that a strong classical light source modulated in repetitive pulses would produce the same resonances as the phaselocked laser beam : for each frequency of the source side bands appear with spectrum and phases comparable to that of the multimode laser. The summations over the incoherent spectrum of the source (summation over $S$ and $\delta$ in (70)) do not modify the Zeeman coherence effect resonances.

VI. Standing wave. - All our experiments have been performed with the cell inside the laser cavity. Therefore it was necessary to study the effect of using a standing wave instead of a travelling wave. Here we shall only summarize the interesting results (for more details, see [4]).

All along the calculation we must take into account two waves of opposite $\mathbf{k}$ for each mode. As we are concerned with terms of slow spatial modulation $\left(\left|\mathbf{k}_{v}-\mathbf{k}_{\mu}+\mathbf{k}_{\lambda}-\mathbf{k}_{\boldsymbol{\kappa}}\right| \ll\left|\mathbf{k}_{v}\right|\right)$, at fourth order we have to consider the three cases of Table II. The case A corresponds to twice the previously studied travelling wave problem. The case $\mathrm{C}$, which introduces $v$ dependence in the Zeeman factor (terms like

$$
\left.\left(\Gamma_{b}^{\prime}(2)+i\left(\omega_{v}-\omega_{\mu}+2 \omega_{b}+2 \mathrm{k} v\right)\right)^{-1}\right)
$$

fortunately disappears when the integration over $v$ is performed with the Doppler approximation. The physical interpretation is that case $\mathrm{C}$ corresponds, at second order, to terms spatially modulated by $\cos \left(\left(k_{\nu}+k_{\mu}\right) r\right)$ and which are averaged to zero by atomic motion (mean free path $>\lambda / 2$ ).

\begin{tabular}{|c|c|c|c|c|}
\hline Order : & 1 & 2 & 3 & 4 \\
\hline Modes : & $v$ & $\mu$ & $\lambda$ & $\kappa$ \\
\hline - & 一 & - & - & - \\
\hline \multirow{2}{*}{ A } & $\rightarrow$ & $\rightarrow$ & $\rightarrow$ & \\
\hline & $\leftarrow$ & $\leftarrow$ & $\leftarrow$ & $\leftarrow$ \\
\hline \multirow{2}{*}{ B } & $\rightarrow$ & $\rightarrow$ & $\leftarrow$ & $\leftarrow$ \\
\hline & $\leftarrow$ & $\leftarrow$ & $\rightarrow$ & $\rightarrow$ \\
\hline \multirow{2}{*}{$\mathrm{C}$} & $\rightarrow$ & $\leftarrow$ & $\rightarrow$ & $\leftarrow$ \\
\hline & $\leftarrow$ & $\rightarrow$ & $\leftarrow$ & \\
\hline
\end{tabular}

\section{TABLE II}

The 6 possible choices for the propagation directions of waves form 3 symmetrical pairs.

The case B produces interesting new terms which all have the spatial dependance

$$
\cos 2\left(k_{v}-k_{\mu}\right) r=\cos 2 \pi(v-\mu) \frac{r}{L} .
$$

They can be deduced from the travelling wave result (44) by substituting

$$
\begin{aligned}
X\left(\frac { 1 } { 2 } \left(\delta_{v}-\right.\right. & \left.\left.\delta_{\kappa}+\left(q_{1}+q_{4}\right) \omega_{b}\right)\right) \times \\
& \times\left(2 \Gamma_{a b}^{\prime}+i\left(\delta_{v}+\delta_{\kappa}+\left(q_{1}-q_{4}\right) \omega_{b}\right)\right)^{-1}
\end{aligned}
$$

to the factors

$$
\begin{aligned}
X\left(\frac { 1 } { 2 } \left[\delta_{\mu}+\right.\right. & \left.\left.\delta_{\kappa}-\left(q_{2}+q_{4}\right) \omega_{b}\right]\right) \times \\
& \times\left(2 \Gamma_{a b}^{\prime}+i\left(\delta_{\kappa}-\delta_{\mu}+\left(q_{2}-q_{4}\right) \omega_{b}\right)\right)^{-1}
\end{aligned}
$$

This substitution in the optical coincidence factor means that the first pair of modes $\left(v_{q_{1}}, \mu_{-q_{2}}\right)$ and the second pair $\left(\lambda_{q_{3}}, \mu_{-q_{4}}\right)$, shifted by Zeeman effect, must be symmetric with respect to the atomic central frequency, in order to interact with atoms of the same 
velocity (the Doppler shift are of opposite sign). Therefore the optical coincidence factor depends on the absolute frequency of modes. On the other hand, the Zeeman factor $\left(\Gamma_{b}^{\prime}(2)+i\left(\omega_{v}-\omega_{\mu}+Q \omega_{b}\right)\right)^{-1}$ is the same in cases A and B : this is obvious, as these two cases do not differ up to the second order (Table II). Finally, the contribution of atoms whose velocity has changed is exactly the same in cases A and B (except the factor $\cos 2\left(k_{v}-k_{\mu}\right) r$ ). This is evident with the model used, as one atom whose velocity has changed has exactly the same probability to have the final velocities $v$ or $-v$.

When the laser is $\sigma$ linearly polarized, the population effect (case $\mathrm{b}$ in Table I) produces resonances of the form $\left(2 \Gamma_{a b}^{\prime}+i\left(\delta_{v}+\delta_{\kappa}+2 \omega_{b}\right)\right)^{-1}$, each time the Zeeman splitting is such as the $\sigma^{+}$(or $\sigma^{-}$) central frequency is at equal distance between two modes : the position of these resonances depends on the mode frequency. For a monomode laser, this term produces a magnetically scanned «Lamp dip». The case a of Table I produces terms of the form

$$
\left(2 \Gamma_{a b}^{\prime}+i\left(\delta_{v}+\delta_{\kappa}\right)\right)^{-1}
$$

which depends only on modes frequency. When modes are very close and numerous $\left(\Gamma_{a b}^{\prime} \gtrsim \Delta \omega, N \Delta \omega \sim \Delta v\right)$ all $P$. E. resonances overlap and disappear exactly as for a travelling wave.

The ZCE terms (case $\mathrm{c}$ in Table I) have two denominators,

$$
\begin{aligned}
\left(\Gamma_{b}^{\prime}(2)+i\left(\delta_{v}-\delta_{\mu}+2 \omega_{b}\right)\right)^{-1} \times \\
\left(2 \Gamma_{a b}^{\prime}+i\left(\delta_{v}+\delta_{\kappa}+2 \omega_{b}\right)\right)^{-1},
\end{aligned}
$$

which cannot be simultaneously resonant, except if modes are symmetrical with respect to the atomic frequency $\omega$. When modes are close $\left(\Gamma_{a b}^{\prime} \gtrsim \Delta \omega\right)$ the resonance of the optical factor are not very sharp and the result is comparable to that of the travelling wave case. If it is possible to apply the analytical approximation (condition (69)), it is easy to show that cases A and $B$ give the same result when the modes are phase locked : then the formula (71) has only to be multiply by $(1+\cos 2 \pi p r / L)$. If the cell is put near the mirror $(\cos 2 \pi p r / L \sim 1)$ all resonances are multiplied by 2 , but resonances $p \neq 0$ cancel in some positions in the laser cavity [31].

On the other hand, when modes are free running, the contribution of case B is very small, except for the $p=0$ resonance which is unaffected, so that (73) can be kept. Indeed as the phase relation imposes $v=\kappa$ (and $\mu=\lambda$ ), only a few modes can fulfill the two resonances conditions : $2 \delta_{v} \simeq-2 \omega_{b}=\delta_{v}-\delta_{\mu}$.
Therefore with a standing wave, the contrast between the $p=0$ resonance and the others is twice that obtained with a travelling wave.

VII. General case $\left(J_{a}\right.$ and $\left.J_{b} \neq 0\right)$. Measurement of Landé $g$ factors. - When $J_{a}=0$ and $J_{b}=1$, it is easy to measure the Lande factor of the $b$ level by simultaneously measuring the position of resonances in magnetic field scale, and the frequency of the beat notes between modes (Fig. 1). In the general case, it is not possible to say, without calculation, to what level the observed resonances must be attributed.

As has been seen in paragraphe III.1, the general case is not easy to solve due to the coupling between eq. $(22 c)$. The approximation made in reference [13] (neglecting $\omega_{a}-\omega_{b}$ in $(22 c)$ ) allows us to calculate second order terms such as Hanle effect; it allows too the interpretation of saturation behaviour of the previous paragraphs, but it is a priori bad to determine the exact position of resonances, as the approximation is made on the Zeeman splitting itself. On the other hand, with the assumption that all relaxation rates of optical quantities are equal $\left(G_{a b}(k)=G\right)$, it is possible to take into account the exact Zeeman structure of the laser line, by using the basis $\left|J_{\alpha} M_{\alpha}\right\rangle$ instead of the tensorial operators ${ }_{a b} T_{Q}^{k}$ for optical coherence ${ }_{a b} \rho$ (and keeping ${ }_{\alpha} T_{Q}^{k}$ for atomic quantities ${ }_{\alpha} \rho$ ): with this basis, each matrix element $<J_{a} M_{a}|\rho| J_{b} M_{b}>$ has its own frequency

$$
\left(\omega+M_{b} \omega_{b}-M_{a} \omega_{a}\right)
$$

and odd order eq. $(22 c)$ are not coupled. We shall just give some results of this rather involved calculation [4].

As is suggested intuitively, each optical coincidence factor in the previous calculation must be replaced by a sum of several terms resonant for different magnetic fields. Indeed each coincidence in a normal Zeeman pattern (say for instance $\left(\mu, \sigma^{+}\right) \leftrightarrow\left(\kappa, \sigma^{-}\right)$) is replaced by several coincidences (each $\left(\mu, \sigma_{n}^{+}\right)$component with each $\left.\left(\kappa, \sigma_{m}^{-}\right)\right)$. On the other hand, the Zeeman factors keep the same form, but new terms appear, containing $\left[\Gamma_{a}(k)+i\left(\omega_{v}-\omega_{\mu}+Q \omega_{a}\right)\right]^{-1}$, to take into account tensorial quantities in the $a$ level.

When modes are close $\left(\Gamma_{a b}^{\prime} \gtrsim \Delta \omega\right)$ PE resonances disappear exactly as in the $J_{a}=0$ case, and similarly the Zeeman factors completely determine position and shape of the ZCE resonances. Finally, in that case, the two methods of calculation give the same result, which is composed only of two sets of resonances corresponding to the two levels. The observed saturation signal is given (for very close modes) by :

$$
\begin{aligned}
S_{p} \text { (locked) }=2 n\left|P_{a b}\right|^{4} \Delta \omega^{-2}\left\{\left(C_{b} \mathcal{L}_{b}^{\prime}(p)+C_{a} \tilde{\mathcal{L}}_{a}^{\prime}(p)\right) \pi \int I(\delta) I(\delta+P) X\left(\delta+\frac{P}{2}\right) \frac{\mathrm{d} \delta}{\Delta v}+\right. \\
\left.+\left[C_{b}\left(\mathcal{L}_{b}(p)-\mathcal{L}_{b}^{\prime}(p)\right)+C_{a}\left(\tilde{\mathcal{L}}_{a}(p)-\tilde{\mathcal{L}}_{a}^{\prime}(p)\right)\right]\left(\int \sqrt{I(\delta) I(\delta+P)} X\left(\delta+\frac{P}{2}\right) \frac{\mathrm{d} \delta}{\Delta v}\right)^{2}\right\}
\end{aligned}
$$


where $\mathfrak{L}_{b}(p)$ and $\mathfrak{L}_{b}^{\prime}(p)$ are the Lorentzian resonances from the $b$ level $(72)$ and $\widetilde{\mathfrak{L}}_{a}(p)$ and $\widetilde{\mathfrak{L}}_{a}^{\prime}(p)$ are the resonances from the $a$ level distorted by spontaneous emission from $b$ level :

$$
\begin{aligned}
\tilde{\mathfrak{L}}_{a}(p)=\Gamma_{a}(2)\left(\Gamma_{a}^{2}(2)+\left(P+2 \omega_{a}\right)^{2}\right)^{-1}- & \\
& \quad-\gamma_{b a} \mathcal{A}(1,2) \operatorname{Re}\left\{\left(\Gamma_{a}(2)+i\left(P+2 \omega_{a}\right)\right)^{-1}\left(\Gamma_{b}(2)+i\left(P+2 \omega_{b}\right)\right)^{-1}\right\} .
\end{aligned}
$$

The transfer coefficient $\mathcal{A}$ and the relative height of resonances, $C_{a}$ and $C_{b}$, are given in appendix as a function of the $J$ values of the levels. It is important to notice that $C_{a}$ and $C_{b}$ depend on the fluorescence lines which are used to observe the resonances, and on their polarization. This is due to the fact that the observed signal is a linear combination of the longitudinal quantites ${ }_{\alpha}^{(4)} \rho_{0}^{k}$ which are not proportional to each other in the general case : the coefficients of the combination are determined by the method of detection.

In many cases the resonances from $a$ and $b$ are not resolved well enough to allow separate measurement of the $g$-factors. Nevertheless, comparing the values of $C_{a} / \Gamma_{a}(2)$ and $C_{b} / \Gamma_{b}(2)$, it is possible to show that, in some cases, the set of resonances from one of the levels has a negligible amplitude, compared to the resonances from the other level. Therefore, it is possible to measure the Lande factor of the dominant level.

For a $J_{b}=1, J_{a}=2$ laser line, the $J_{a}=2$ level appears to be the dominant level in all experimental cases. In fact, if $\Gamma_{a}(2) \sim \Gamma_{b}(2), C_{a}$ is approximately 21 times $C_{b}$ (cf. Appendix). Although it has been demonstrated in that case [10] that fourth order perturbation theory rapidly diverges from the exact solution when the laser intensity is not very small, the above result has been clearly shown experimentally [32] : the resonances spacings, observed on the $6096 \AA$ line $\left(2 p_{4} \rightarrow 1 s_{4}\right)$, are exactly the same with the $6328 \AA\left(3 s_{2} \rightarrow 2 p_{4}\right)$ or $1.15 \mu\left(2 s_{2}-2 p_{4}\right)$ iaser lines and correspond to the $2 p_{4}$ Landé factor (Fig. 7). However, the upper levels have different Landé factor $\left(g_{3 s_{2}}=1.295 ; g_{2 s_{2}}=1.224\right)$. The numerical estimate of $C_{a}$ and $C_{b}$ shows that the systematic error on the measured value of the $2 p_{4}$ Lande factor is less than 0.5 per cent (laser $1.15 \mu$, fluorescence $6096 \AA$ ) but can be reduced to $10^{-4}$ with a correct choice for pressure and for the fluorescence line.

For a $J_{a}=1, J_{b}=1$ laser line the $C_{a}$ and $C_{b}$ values strongly depend on the polarization of the chosen fluorescence line and on the $J_{g}$ value of the lower level, $g$, of this fluorescence line (cf. Appendix). If there is only one relaxation rate for each level $\left(\Gamma_{\alpha}(0)=\Gamma_{\alpha}(2)\right)$ and if the spontaneous emission on the laser line is negligible, it appears, in some cases, that either $C_{a}$, either $C_{b}$, vanishes, so that it is very easy to measure Landé factors. These interesting cases can be easily found graphically (Fig. 8). As there is then only one relaxation rate for each level, there is no coupling between Zeeman sublevels and it is possible to discuss in the basis $\left|J_{\alpha} M_{\alpha}\right\rangle$ (instead

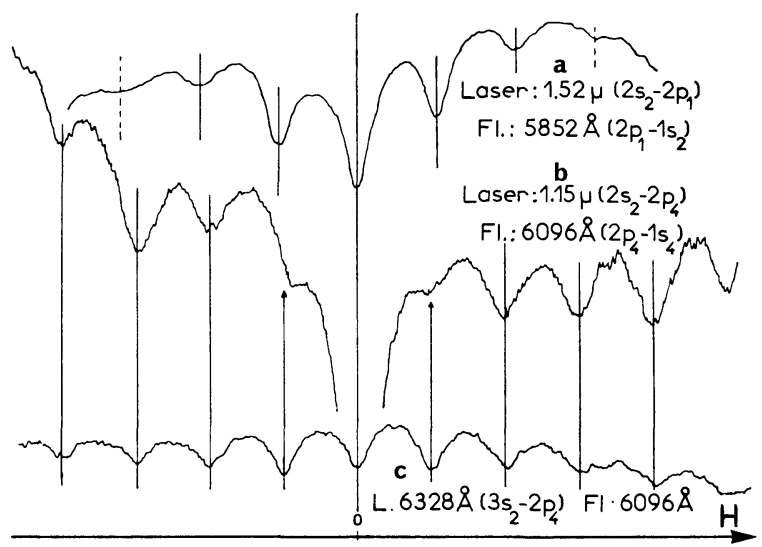

FIG. 7. - The two lower records show saturation resonances obtained with the $J_{b}=2 \leftrightarrow J_{a}=1$ laser lines $\lambda=6328 \AA$ and $\lambda=1.15 \mu$ : the resonance spacing is exactly the same and corresponds to the Lande factor of their common lower level $\left(2 p_{4}, J=2\right)$. As a comparison the upper records, obtained with the $\lambda=1.52 \mu$ laser line $(J=1 \rightarrow J=0)$ shows the resonances which correspond to the Landé factor of the $2 s_{2}$ level (upper level of the $1.15 \mu$ line).

of the ${ }_{\alpha} T_{Q}^{k}$ basis). Consider, for instance, the case $\beta$ in figure 8 (detection of the $\pi$ component of a $a \rightarrow g$ line with $\left.J_{g}=0\right)$ : we detect only the population of the $M_{a}=0$ sublevel which is coupled only to the coherence $M_{b}=-1 \leftrightarrow M_{b}=+1$ by the $\sigma$ polarized laser beam. Therefore saturation resonances observed with this configuration are due only to the $b$ level. Let us notice that this particular result is true at all orders of perturbation, as the three concerned sublevels are never coupled to the three others for any number of interactions with the laser. On the other hand, when $\Gamma_{\alpha}(2) \neq \Gamma_{\alpha}(0)$, the destruction of alignment by relaxation processes introduces a coupling between Zeeman sublevels and the previous discussion fails.
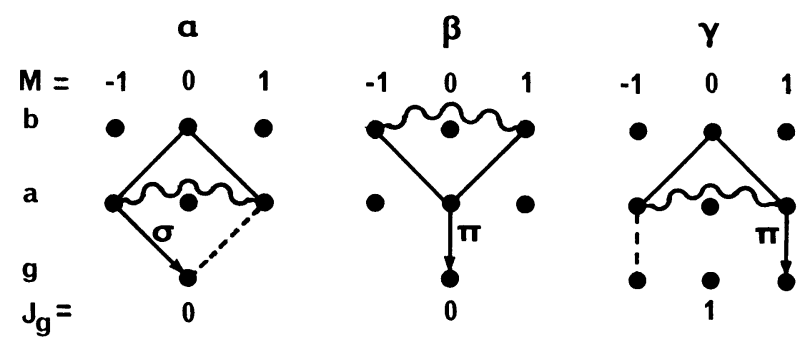

FIG. 8. $-J_{a}=1, J_{b}=1$ : graphical illustration of the three cases for which it is possible to attribute the observed saturation resonances to only one level, if there is no coupling between the Zeeman sublevels by relaxation processes. Dots represent the Zeeman sublevels, full lines the laser interaction $\left(\sigma^{+}\right.$or $\left.\sigma^{-}\right)$, arrows the fluorescence lines and the waved lines represent the Zeeman coherence which is detected on the saturation signal. 
An experimental verification of these results has been performed with the $6401 \AA$ laser line of $\mathrm{Ne}$ $\left(3 s_{2}, J_{b}=1 \rightarrow 2 p_{2}, J_{a}=1\right)$. In a first experiment we have used the fluorescence lines $6351 \AA$ ( $\left.3 s_{2}, J_{b}=1 \rightarrow 2 p_{3}, J_{g}=0\right)$ and $5434 \AA\left(3 s_{2}\right.$, $\left.J_{b}=1 \rightarrow 2 p_{10}, J_{g}=1\right)$ both detected with a $\pi$ analyser. According to the diagrams of figure 8, the $6351 \AA$ is assumed to provide the $2 p_{2}$ Landé factor and the $5434 \AA$ the $3 s_{2}$ Landé factor. Actually these experiments were performed with pure Neon at a pressure of 1.5 torr so that $\Gamma_{b}(2)(7.75 \mathrm{MHz})$ was approximately twice $\Gamma_{b}(0)(3.85 \mathrm{MHz})$ due to collisions and trapping of the resonance line $3 s_{2} \rightarrow 1 p_{0}$. Therefore the calculated values of $C_{a}$ and $C_{b}$ show a considerable mixing of both kinds of resonances on each fluorescence line. This conclusion is well verified: 1) The spacing between resonances on the $5434 \AA$ lines does not correspond exactly to the $3 s_{2}$ level $(33.15 \pm 0.1 \mathrm{~mm}$ instead of $33.56 \pm 0.1 \mathrm{~mm}$ with the $7305 \AA$ laser line $-3 s_{2}, J=1 \rightarrow 2 p_{1}, J=0$ - with the same apparatus and the same mode spacing). 2) The measured ratio $g_{3 p_{2}} / g_{3 s_{2}}$ is smaller than the expected one from tables [33] (1.028 $5 \pm 0.0018$ instead of $1.0348 \pm 0.006)$.

In a second set of measurements the $6163 \AA$ $\left(2 p_{2}, J_{a}=1 \rightarrow 1 s_{3}, J_{g}=0\right)$ and the $6599 \AA\left(2 p_{2}\right.$, $\left.J_{a}=1 \rightarrow 1 s_{2}, J_{g}=1\right)$ fluorescence lines were used ( $\pi$ polarization) with a cell containing 1 torr of a 9 : 1, He : Ne mixture (Fig. 9). As in those conditions $\Gamma_{a}(0)(9.45 \mathrm{MHz})$ and $\Gamma_{a}(2)(10.6 \mathrm{MHz})$ are not very different from each other and as the spontaneous emission on the $6401 \AA$ line is weak, the calculation of $C_{a}$ and $C_{b}$ shows that the diagrams of figure 8 can be used with a good precision: the $6163 \AA$ line $\left(J_{g}=0\right)$ gives the $3 s_{2}$ Landé factor (estimated residual shift: $+3 \times 10^{-4}$ ) and the $6599 \AA$ line $\left(J_{g}=1\right)$ gives the $2 p_{2}$ Landé factor (estimated shift $-2 \times 10^{-3}$ ). The measured

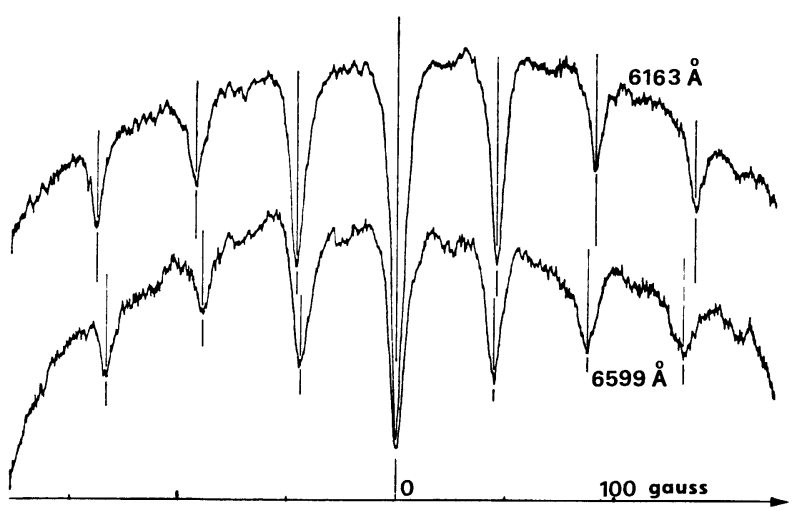

FIG. 9. - Laser $6401 \AA\left(3 s_{2}, J=1 \rightarrow 2 p_{2}, J=1\right)$. The two curves are simultaneously record with two different fluorescence lines $\left(\pi\right.$ polarization). The $6163 \AA$ line $\left(2 p_{2}, J=1 \rightarrow 1 s_{3}\right.$, $J=0$ ) gives the Landé factor of the $3 s_{2}$ level (case $\beta$ on Fig. 8). The $6599 \AA$ line $\left(2 p_{2}, J=1 \rightarrow 1 s_{2}, J=1\right)$ gives the $2 p_{2}$ Landé factor (case $\gamma$ on Fig. 8). These records are obtained with $\Delta \omega=166 \mathrm{MHz}$ and with experimental conditions for which $\Gamma_{2 p_{2}}(0) \simeq \Gamma_{2 p_{2}}(2)$ (within $10 \%$ ). $g_{2 p_{2}} / g_{3 s_{2}}$ ratio $(1.0357 \pm 0.0016)$ is in good agreement with table data $(1.0348 \pm 0.006)$. In spite of a very poor magnetic field calibration in this last experiment the measured absolute $g$ values are in good agreement with tables $\left(g_{3 s_{2}}=1.290 \pm 0.014\right.$ instead of $1.295 \pm 0.005 ; g_{2 p_{2}}=1.336 \pm 0.016$ instead of $1.340 \pm 0.003$ ).

On the basis of the above discussion we have performed measurement of Landé $g$ factors for many Neon levels [4], [32] (2 $p_{4}$ with $6328 \AA$ laser, $3 s_{2}$ with $7305 \AA, 2 s_{2}$ with $1.52 \mu, 3 p_{4}$ with $3.39 \mu$, $2 p_{2}$ with $6401 \AA$ ). The values obtained are all in good agreement with other authors' results but the precision $\left(\sim 10^{-2}\right)$ is limited by the magnetic field calibration (our coils are too small to be calibrated with proton resonances). On the contrary, as the distance between resonances on the recorder can be measured with a good precision $\left(3 \times 10^{-3}\right)$ we had used this method to calibrate more accurately our magnetic field for other experiments such as Hanle effect (a direct calibration of the recorder in $\mathrm{MHz}$, for each level, allows the measurement of relaxation times from Hanle effect without any need of the Landé factor).

With coils specially built for high homogeneity, one can expect a precision of $3 \times 10^{-3}$ on Landé $g$ factor measurements, with the above self mode locking method. Further improvement could be achieved by using an intra cavity modulator to produce mode-locking with higher laser intensity (better signal to noise ratio, higher number of modes). The ultimate precision, determined by the resonances width can be expected to be better than $10^{-3}$ as for magnetic resonance experiments, but at this extreme precision the mixing of the two levels must be studied more carefully.

VIII. Conclusion. - The relative magnitude of the mode spacing $\Delta \omega$ and of the hole width (or optical relaxation rate $\Gamma_{a b}^{\prime}$ ) appears to be of great importance in determining the behaviour and the shape of saturation resonances. We have shown that the condition $\Gamma_{a b}^{\prime} \gtrsim \Delta \omega$ which allows several modes to interact with the same atoms permits us to understand our experimental observations and particularly the effect of the relative phase of modes on the resonances in non-zero magnetic field. The resonances of saturation are due to the Zeeman coherence ; they are the manifestation of the Hanle effect and of the modulation resonances observed on the linear response. When the modes are numerous enough, the shape of resonances tends to the Hanle effect shape. On the other hand all resonances due to hole burning (or population effects) overlap and disappear.

The influence of atomic velocity diffusion has been shown to disappear when the modes are numerous and phase locked. In other cases the experimental investigation of the shape of resonances would be of interest to study these velocity diffusion processes. 
Finally we have demonstrated the possibility of measuring Landé $g$ factors in some cases.

We did not try to verify experimentally the calculated shape of resonances as our perturbation method is valid only for vanishing laser intensities. Ducloy [10] has succeeded to solve this problem for the resonance in zero magnetic field in some special cases

$$
\left(J_{b}=1 \leftrightarrow J_{a}=0 ; \quad J_{b}=1 \leftrightarrow J_{a}=1\right)
$$

\section{APPENDIX}

In (75) the relative height of resonances from $a$ and $b$ levels is determined by the coefficients $C_{a}$ and $C_{b}$ defined by

$$
\begin{aligned}
C_{a} & =-\sum_{k^{\prime}}(-)^{k^{\prime}} a\left(k^{\prime}\right) \sqrt{5}\left(\begin{array}{ccc}
2 & 1 & k^{\prime} \\
2 & -1 & -1
\end{array}\right) \quad\left\{\begin{array}{lll}
1 & 1 & 2 \\
J_{a} & J_{a} & J_{b}
\end{array}\right\}\left\{\begin{array}{lll}
2 & 1 & k^{\prime} \\
J_{b} & J_{a} & J_{a}
\end{array}\right\} \\
C_{b} & =\sum_{k^{\prime}} a\left(k^{\prime}\right) \sqrt{5}\left(\begin{array}{ccc}
2 & 1 & k^{\prime} \\
2 & -1 & -1
\end{array}\right)\left\{\begin{array}{lll}
1 & 1 & 2 \\
J_{b} & J_{b} & J_{a}
\end{array}\right\}\left\{\begin{array}{lll}
2 & 1 & k^{\prime} \\
J_{a} & J_{b} & J_{b}
\end{array}\right\}
\end{aligned}
$$

where $k^{\prime}$ is the tensorial order of optical quantities at third order of perturbation. If the detection is made with $a b \rightarrow g$ line, $a\left(k^{\prime}\right)$ is :

$$
\begin{aligned}
a_{b}\left(k^{\prime}\right)=\frac{\delta_{1, k^{\prime}}}{3\left(2 J_{b}+1\right) \Gamma_{b}(0)}-\sqrt{\frac{10}{3} \frac{\left(2 k^{\prime}+1\right) \alpha}{\Gamma_{b}(2)}(-)^{2 J_{b}+J_{a}+J_{y}}} \times \\
\quad \times\left\{\begin{array}{lll}
k^{\prime} & 1 & 2 \\
J_{b} & J_{b} & J_{a}
\end{array}\right\}\left\{\begin{array}{rlll}
1 & 1 & 2 \\
J_{b} & J_{b} & J_{g}
\end{array}\right\}\left(\begin{array}{rrr}
k^{\prime} & 1 & 2 \\
1 & -1 & 0
\end{array}\right) .
\end{aligned}
$$

If the detection is made with a $a \rightarrow g$ line $a\left(k^{\prime}\right)$ is :

$$
\begin{aligned}
& a_{a}\left(k^{\prime}\right)=\frac{\delta_{1,},}{3\left(2 J_{a}+1\right)} \overline{\Gamma_{a}(0)}\left(1-\frac{\gamma_{b a}}{\Gamma_{b}(0)}\right)-\sqrt{\frac{10}{3}} \frac{\left(2 k^{\prime}+1\right) \alpha}{\Gamma_{a}(2)}\left(1-\frac{\gamma_{b a} A\left(k^{\prime}, 2\right)}{\Gamma_{b}(2)}\right)(-)^{k^{\prime}+2 J_{a}+J_{b}+J_{g}} \times \\
& \left.\times\left\{\begin{array}{ccc}
k^{\prime} & 1 & 2 \\
J_{a} & J_{a} & J_{b}
\end{array}\right\}\left\{\begin{array}{ccc}
1 & 1 & 2 \\
J_{a} & J_{a} & J_{g}
\end{array}\right\} \quad \begin{array}{rrr}
k^{\prime} & 1 & 2 \\
1 & -1 & 0
\end{array}\right)
\end{aligned}
$$

in (79) and (80) $\alpha=1$ for a $\pi$-detection and $\alpha=-1 / 2$ for a $\sigma$-detection. The transfer coefficient $\mathcal{A}$ which also occurs in (76) is given by

$$
\mathcal{A}\left(k^{\prime}, k\right)=(-)^{J_{a}+J_{b}+k^{\prime}}\left(2 J_{b}+1\right)\left\{\begin{array}{lll}
k & J_{b} & J_{b} \\
1 & J_{a} & J_{a}
\end{array}\right\} \quad\left\{\begin{array}{lll}
k^{\prime} & 1 & k \\
J_{b} & J_{b} & J_{a}
\end{array}\right\} \quad\left\{\begin{array}{ccc}
k^{\prime} & 1 & k \\
J_{a} & J_{b} & J_{b}
\end{array}\right\}^{-1} .
$$

Finally we express $C_{a}$ and $C_{b}$ in two useful cases:

$\left.\underline{J_{b}=1, J_{a}=2} .-a\right)$ Fluorescence line $b \rightarrow g:$

$$
\begin{aligned}
& C_{b}(b \rightarrow g)=\frac{1}{8100}\left[\frac{1}{\Gamma_{b}(0)}-\frac{\alpha \beta}{\Gamma_{b}(2)}\right] \\
& C_{a}(b \rightarrow g)=\frac{1}{2700}\left[\frac{7}{\Gamma_{b}(0)}+\frac{2 \alpha \beta}{\Gamma_{b}(2)}\right]
\end{aligned}
$$

with $\beta=1,-1 / 2$ or $1 / 10$ respectively for $J_{g}=0,1$ or 2

b) Fluorescence line $a \rightarrow g$ :

$$
\begin{aligned}
& C_{b}(a \rightarrow g)=\frac{-1}{13500}\left[\frac{1}{\Gamma_{a}(0)}\left(1-\frac{\gamma_{b a}}{\Gamma_{b}(0)}\right)+\frac{\alpha \beta^{\prime}}{\Gamma_{a}(2)}\left(1+\frac{7 \gamma_{b a}}{20 \Gamma_{b}(2)}\right)\right] \\
& C_{a}(a \rightarrow g)=\frac{-1}{4500}\left[\frac{7}{\Gamma_{a}(0)}\left(1-\frac{\gamma_{b a}}{\Gamma_{b}(0)}\right)+\frac{\alpha \beta^{\prime}}{\Gamma_{a}(2)}\left(\frac{3}{2}-\frac{7 \gamma_{b a}}{10 \Gamma_{b}(2)}\right)\right]
\end{aligned}
$$

with $\beta^{\prime}=1,-1$ or $2 / 7$ if $J_{q}=1,2$ or 3 . 
$\underline{J_{a}=1, J_{b}=2}$.

$$
\begin{aligned}
& C_{b}(b \rightarrow g)=\frac{1}{324}\left(\frac{1}{\Gamma_{b}(0)}-\frac{\alpha \beta}{\Gamma_{b}(2)}\right) \\
& C_{a}(b \rightarrow g)=\frac{1}{324}\left(\frac{1}{\Gamma_{b}(0)}+\frac{2 \alpha \beta}{\Gamma_{b}(2)}\right) \\
& C_{b}(a \rightarrow g)=\frac{-1}{324}\left[\frac{1}{\Gamma_{a}(0)}\left(1-\frac{\gamma_{b a}}{\Gamma_{b}(0)}\right)+\frac{2 \alpha \beta}{\Gamma_{a}(2)}\left(1-\frac{\gamma_{b a}}{4 \Gamma_{b}(2)}\right)\right] \\
& C_{a}(a \rightarrow g)=\frac{-1}{324}\left[\frac{1}{\Gamma_{a}(0)}\left(1-\frac{\gamma_{b a}}{\Gamma_{b}(0)}\right)-\frac{\alpha \beta}{\Gamma_{a}(2)}\left(1-\frac{\gamma_{b a}}{\Gamma_{b}(2)}\right)\right]
\end{aligned}
$$

with $\beta=1,-1 / 2$ or $1 / 10$ if $J_{g}=0,1$ or 2 .

If $\Gamma_{b}(0)=\Gamma_{b}(2)$, for $J_{g}=0, C_{b}(b \rightarrow g)=0$ with a $\pi$ detection and $C_{a}(b \rightarrow g)=0$ with a $\sigma$ detection. For $J_{g}=1$ and a $\pi$ detection $C_{a}(b \rightarrow g)=0$. If $\Gamma_{a}(0)=\Gamma_{a}(2)$ and if the spontaneous emission on the laser line is negligible $\left(\gamma_{b a} \simeq 0\right)$ we obtain the symmetrical cases which correspond to the figure 8 .

\section{References and Notes}

[1] Decomps (B.) and Dumont (M.), J. Physique, 1968, 29, 443 IEEE J. of Quant. Electronics, 1968, QE 4, 916.

[2] Decomps (B.) and Dumont (M.), C. R. Acad. Sci., Paris, 1966, 262B, 1520

[3] Fork (R. L.), Hargrove (L. E.) and Pollack (M. A.), Phys. Rev. Lett., 1964, 12, 705.

[4] Dumont (M.), Thèse, Paris, 1971 (Microfilm CNRS $n^{\circ}$ AO 5608)

[5] Bennett (W. R.), Phys. Rev., 1962, 126, 580.

[6] Sмith (P. W.), J. of Appl. Phys., 1966, 37, 2089.

HäNSCH (T.) and TOSCHEK (P.), IEEE J. of Quant. Electronics, 1968, QE 4, 530 and 1969, QE 5, 61.

[7] Holtz (H.), Phys. Rev. Lett., 1967, 19, 1275 and 1968, 20, 410.

[8] Feld (M. S.) and Javan (A.), Phys. Rev., 1969, 177, 540.

[9] Lamb (W. E.), Phys. Rev., 1964, 134, A 1429.

[10] Ducloy (M.), Opt. Com., 1971, 3, 205 ; Calculation to be published.

Datchary (J.), and Ducloy (M.), C. R. Acad. Sci., Paris, 1972, 274B, 337.

[11] Rautian (S. G.), JETP, 1966, 51, 1176, Soviet Phys. JETP, 1967, 24, 788.

Rautian (S. G.) and Sobel'man (I. I.), Sov. Phys. Uspekhi, 1967, 9, 701.

[12] GyorfFy (B. L.), Borenstein (M.) and LAMB (W. E.), Phys. Rev., 1968, 169, 340.

[13] Dumont (M.) and Decomps (B.), J. Physique, 1968, 29, 181.

[14] Cohen-Tannoudji (C.), Thèse, Paris, 1962 ; Ann. de Phys., $1962,7,423$ et 469.

[15] Fano (U.), Rev. of Mod. Phys., 1957, 29, 74.

[16] Omont (A.), J. Physique, 1965, 26, 26.

[17] We use the normalization: $\operatorname{Tr}\left({ }_{\alpha \beta} T_{Q \alpha^{\prime} \beta^{\prime}}^{k} T_{Q^{\prime}}^{k^{\prime}}\right)=$ $\delta_{\alpha \alpha^{\prime}} \delta_{\beta \beta^{\prime}}, \delta_{k k^{\prime}}, \delta_{Q Q^{\prime}}$ and the reduced matrix element is $\left\langle J_{\alpha}\left\|_{\alpha^{\prime} \beta^{\prime}} T_{Q}^{k}\right\| J_{\beta}\right\rangle=\delta_{\alpha \alpha^{\prime}} \delta_{\beta \beta^{\prime}} \sqrt{2 k+1}$.

[18] Carrington (C. G.) and Corney (A.), Opt. Com.., 1969, 1, 115 ; J. of Phys. B, 1971, 4, 849.

[19] Omont (A.), J. Physique, 1965, 26, 576 ; Thèse, Paris, 1967.

[20] D'Yakonov (M. I.) and Perel (V. I.), Sov. Phys. JETP, 1965, 20, 997.

[21] Saloman (E. B.) and Happer (W.), Phys. Rev., 1966, 144, 7

[22] Lombardi (M.), C. R. Acad. Sci., Paris, 1967, 265, 191 ; J. Physique, 1969, 30, 631 ; Thèse Grenoble, 1970.

[23] When there are many modes $(N \Delta \omega>\Delta v)$ very close to each other $\left(\Delta \omega<\Gamma_{a b}\right)$ and of equal intensity it is easy to see that ${ }_{\alpha} \rho(v)={ }_{\alpha} \bar{\rho} W_{M}(v)$, as is obtained with a classical broad line source; in that case the equations for atomic quantities can be integrated over $v$ before being solved (this is not true for optical quantities). Therefore the classical relaxation rates $\Gamma_{\alpha}(k)$ are reintroduced, whatever the anisotropy is for each particular atomic velocity. This fact justifies the use of the isotropic approximation at second order (for the Hanle effect) but also at fourth order and at all even orders [10], to deal with a multimode laser.

[24] Sмiтh (P. W.) and HäNSCh (T.), Phys. Rev. Lett., 1971, 26, 740 .

[25] Ducloy (M.) and Dumont (M.), C. R. Acad. Sci., Paris, 1968, 266 B, $340 ; J$. Physique, 1970, 31, 419.

[26] Fried (B.) and ConTe (S.), " The Plasma Dispersion Function ", Academic Press, New York, London 1961.

[27] This property is valid for all velocity change models if the isotropy is assumed. If not, it needs (2) $p(v)$ to have the Maxwellian distribution $W_{\mathrm{M}}(v)$ (approximately valid when modes are numerous and close).

[28] DuCloy (M.), to be published : the estimated value of $\Gamma_{2 p_{1}}$ is $8.5 \mathrm{MHz}$.

[29] Cordover (R, H.) and Bonczyx (P. A.), Phys. Rev. Lett., 1969, 188, 696.

[30] The present perturbation calculation shows that resonances of saturation at fourth order tend to have the shape of the Hanle effect at second order. With a calculation valid at all orders, Ducloy has shown [10], for $a J=1 \leftrightarrow J=0$ laser line, that the saturation resonance $(H=0)$ and the Hanle effect keep the same shape even when they are broadened by a high power laser beam (for very close and very numerous modes). This theoretical result is very well verified experimentally.

[31] This spatial modulation of saturation, which appears also on the small $p \neq 0$ PE terms, can be used to interpret the spontaneous mode locking which occurs when the amplifying medium is put in a region of low saturation, or an absorbing medium in a region of high saturation (cf. Smith [34]) We also observed a spontaneous mode locking while the magnetic field was scanned accross the saturation resonances of an absorbing cell near the mirror.

[32] Decomps (B.) and Dumont (M.), C. R. Acad. Sci., Paris, 1967, 265 B, 249.

[33] Moore (Ch.), "Atomic Energy Levels ", NBS, Washington, 1949.

[34] Sмiтh (P. W.), IEEE J. of Quant. Electronics, 1967, QE 3, 627. 